TRANSACTIONS OF THE

AMERICAN MATHEMATICAL SOCIETY

Volume 353, Number 12, Pages 4757-4777

$\mathrm{S} 0002-9947(01) 02815-\mathrm{X}$

Article electronically published on July 25, 2001

\title{
GEOMETRY OF CHAIN COMPLEXES AND OUTER AUTOMORPHISMS UNDER DERIVED EQUIVALENCE
}

\author{
BIRGE HUISGEN-ZIMMERMANN AND MANUEL SAORÍN
}

The authors wish to dedicate this paper to Idun Reiten on the occasion of her sixtieth birthday

\begin{abstract}
The two main theorems proved here are as follows: If $A$ is a finite dimensional algebra over an algebraically closed field, the identity component of the algebraic group of outer automorphisms of $A$ is invariant under derived equivalence. This invariance is obtained as a consequence of the following generalization of a result of Voigt. Namely, given an appropriate geometrization $\mathrm{Comp}_{\mathbf{d}}^{A}$ of the family of finite $A$-module complexes with fixed sequence $\mathbf{d}$ of dimensions and an "almost projective" complex $X \in \mathrm{Comp}_{\mathbf{d}}^{A}$, there exists a canonical vector space embedding

$$
T_{X}\left(\operatorname{Comp}_{\mathbf{d}}^{A}\right) / T_{X}(G . X) \longrightarrow \operatorname{Hom}_{D^{b}(A-\operatorname{Mod})}(X, X[1])
$$

where $G$ is the pertinent product of general linear groups acting on $\operatorname{Comp}_{\mathbf{d}}^{A}$, tangent spaces at $X$ are denoted by $T_{X}(-)$, and $X$ is identified with its image in the derived category $D^{b}(A$-Mod).
\end{abstract}

\section{INTRODUCTION}

One of our primary goals is to prove that the identity component $\operatorname{Out}(A)^{0}$ of the outer automorphism group of a finite dimensional algebra $A$ is invariant under derived equivalence; here we assume that the base field $K$ of $A$ is algebraically closed. This generalizes a result of Brauer (see [13]), guaranteeing that $\operatorname{Out}(A)^{0}$ is Morita invariant, as well as the next step beyond Brauer's result, which established tilting-cotilting invariance of $\operatorname{Out}(A)^{0}$ (the latter is due to Guil Asensio and the second-named author [7]). On the other hand, note that the full outer automorphism group $\operatorname{Out}(A)=\operatorname{Aut}(A) / \operatorname{Inn}(A)$ is not even Morita invariant, in general, and a fortiori, derived invariance fails for the group $\operatorname{Aut}(A)$ of all algebra automorphisms of $A$. Our invariance theorem was proved independently and with different methods by Rouquier [16].

In his seminal paper [14, Rickard extended results of Happel [8] and ClineParshall-Scott [4], to give an explicit description of the equivalences between derived categories of module categories (strictly speaking, between categories derived

Received by the editors November 6, 2000.

2000 Mathematics Subject Classification. Primary 16E05, 16G10, 16P10, 18E30, 18 G35.

While carrying out this project, the first-named author was partially supported by an NSF grant, and the second-named author by grants from the DGES of Spain and the Fundación 'Séneca' of Murcia. The contents of this article were presented at the Conference on Representations of Algebras at Saõ Paolo in July 1999 and at the Workshop on Interactions between Algebraic Geometry and Noncommutative Algebra at the Mathematical Sciences Research Institute (Berkeley) in February 2000, by the second- and first-named authors, respectively. 
from the triangulated homotopy categories of chain complexes of modules). This characterization opened up the possibility of exhibiting invariants under derived equivalence: Rickard himself showed that the center and the Grothendieck group of $A$ are among them [14], as are the cyclic and Hochschild cohomologies of $A$ (see [9] and [15]). The group $\operatorname{Out}(A)^{0}$, which our final theorem below places on this list, can be viewed as a carrier of homological information as well; indeed, as was first observed by Fröhlich in [6], Out $(A)$ naturally embeds into the Picard group of $A$, that is, into the group of (isomorphism types of) Morita self-equivalences of the category of left $A$-modules. The invariance status of the full Picard group relative to derived equivalence is negative, but self-injective algebras which are derived equivalent share at least the stable Picard group 12 . To round off the picture, we point out that the classical Picard group is an object of natural significance in the context of derived categories, in that it in turn embeds into the 'derived Picard group' $\operatorname{DPic}(A)$, which consists of the isomorphism types of derived self-equivalences of $A$ induced by tilting complexes in $D^{b}\left(\left(A \otimes_{K} A^{\text {op }}\right)\right.$-Mod) (see [17, 20], and [11]). The first of our main results entails that $\operatorname{DPic}(A)$ contains only finitely many $A-A$ tilting complexes of fixed total dimension which are pairwise non-isomorphic when viewed as one-sided complexes over $A$.

This latter result is obtained (in Section 2) as an ingredient of our invariance proof for $\operatorname{Out}(A)^{0}$. We describe it in some detail, since it holds substantial interest in its own right. Suppose $X$ is a point in the classical variety $\operatorname{Mod}_{d}^{A}$ of all $d$-dimensional left $A$-modules. In [19], Voigt exhibited a natural vector space monomorphism

$$
T_{X}\left(\operatorname{Mod}_{d}^{A}\right) / T_{X}\left(\mathrm{GL}_{d} \cdot X\right) \longrightarrow \operatorname{Ext}_{A}^{1}(X, X),
$$

where $T_{X}\left(\operatorname{Mod}_{d}^{A}\right)$ and $T_{X}\left(\mathrm{GL}_{d} . X\right)$ denote the tangent spaces at $X$ to $\operatorname{Mod}_{d}^{A}$ and the $\mathrm{GL}_{d}$-orbit of $X$ in $\operatorname{Mod}_{d}^{A}$, respectively. Here is a sketch of our generalization (Theorem 7): Roughly speaking, it relates tangent spaces of varieties of bounded finite dimensional complexes over $A$ to Hom-groups in the derived category $D^{b}(A$-Mod), whenever $A$ is finite dimensional over an algebraically closed field. More precisely, the geometrization of the $d$-dimensional modules in the framework of $\operatorname{Mod}_{d}^{A}$ can be carried over, in the same spirit, to the complexes of the form $0 \rightarrow X_{m} \rightarrow X_{m-1} \rightarrow$ $\cdots \rightarrow X_{0} \rightarrow 0$ for fixed $m$, where $X_{0}, \ldots, X_{m} \in A$-Mod have prescribed dimensions $d_{0}, \ldots, d_{m}$, respectively. In the predictable manner, this leads to a Zariski-closed subset Compad of

$$
\prod_{0 \leq i \leq m} \operatorname{Hom}_{K}\left(A, \operatorname{End}_{K}\left(K^{d_{i}}\right)\right) \times \prod_{1 \leq i \leq m} \operatorname{Hom}_{K}\left(K^{d_{i}}, K^{d_{i-1}}\right),
$$

where $\mathbf{d}=\left(d_{m}, \ldots, d_{0}\right)$. Moreover, the orbits of the (only plausible) conjugation action of $G=\mathrm{GL}_{d_{0}} \times \cdots \times \mathrm{GL}_{d_{m}}$ on $\mathrm{Comp}_{\mathbf{d}}^{A}$ are in one-to-one correspondence with the isomorphism types of complexes of the described ilk. In this setting, the following is true for any complex $\mathbf{X}$ of finite dimensional left $A$-modules of the above format, with the additional property that all $X_{i}$, except possibly $X_{m}$, are projective over $A$ : If $\mathbf{X}$ is represented by a point $X \in \operatorname{Comp}_{\mathbf{d}}^{A}$, say, then there exists a canonical vector space embedding

$$
T_{X}\left(\operatorname{Comp}_{\mathbf{d}}^{A}\right) / T_{X}(G . X) \longrightarrow \operatorname{Hom}_{D^{b}(A-\operatorname{Mod})}(\mathbf{X}, \mathbf{X}[1]) .
$$

In case all $X_{i}$ are projective, this embedding is actually an isomorphism. Observe that, in $D^{b}(A$-Mod), every finite dimensional left $A$-module coincides with 
a complex of the addressed ilk - to wit, with the stalk complex of the module concentrated in degree $m$ - and hence Voigt's result is retrieved as a special case. Among the consequences, the one instrumental in establishing our result concern$\operatorname{ing} \operatorname{Out}(A)^{0}$ is this: For any finite sequence of non-negative integers, there are only finitely many complexes of projectives with this sequence of dimensions such that $\operatorname{Hom}_{D^{b}(A \text {-Mod })}(\mathbf{X}, \mathbf{X}[1])=0$. So, in particular, we see that $A$ has only finitely many one-sided tilting complexes of prescribed total dimension.

Throughout, $A$ will be a finite dimensional algebra over a field $K$. In the main results of Section 2 and throughout Section $3, K$ will be algebraically closed. Moreover, $A$-Mod, Mod- $A, A$-mod, and mod- $A$ will denote the categories of all left $/ \mathrm{right}$ $A$-modules, and their full subcategories of finite dimensional left/right $A$-modules, respectively.

\section{The geometry of Chain COMPleXes}

Suppose that $A$ is generated by $a_{1}=1, a_{2}, \ldots, a_{s}$ as a $K$-algebra, and recall that the objects in $A$-mod of vector space dimension $d$ are parametrized by the points of the following subvariety of $s \cdot d^{2}$-dimensional affine space:

$$
\begin{aligned}
\operatorname{Mod}_{d}^{A}=\left\{\left(\mathcal{A}_{1}, \ldots, \mathcal{A}_{s}\right) \mid\right. & \text { the } \mathcal{A}_{j} \in \operatorname{End}_{K}\left(K^{d}\right) \text { satisfy } \\
& \text { the same relations as } \left.a_{1}, \ldots, a_{s}\right\} .
\end{aligned}
$$

This variety comes with a canonical morphic $\mathrm{GL}_{d}$-action by conjugation, the orbits of which reflect basis change. So, if one assigns to each point in $\operatorname{Mod}_{d}^{A}$ the corresponding left $A$-module, it is clear that the isomorphism types of $d$-dimensional modules are in one-to-one correspondence with the $\mathrm{GL}_{d}$-orbits in $\operatorname{Mod}_{d}^{A}$. Note that we can identify the points of $\operatorname{Mod}_{d}^{A}$ with points in $\operatorname{Hom}_{K}\left(A, \operatorname{End}_{K}\left(K^{d}\right)\right)$ by assigning to each sequence $\left(\mathcal{A}_{1}, \ldots, \mathcal{A}_{s}\right) \in \operatorname{Mod}_{d}^{A}$ the $K$-algebra homomorphism $A \rightarrow \operatorname{End}_{K}\left(K^{d}\right)$ which sends $a_{j}$ to $\mathcal{A}_{j}$.

We carry this idea over - in the same spirit - to finite chain complexes

$$
\mathbf{X}: \quad 0 \rightarrow X_{m} \stackrel{\partial_{m}}{\longrightarrow} X_{m-1} \stackrel{\partial_{m-1}}{\longrightarrow} \cdots \stackrel{\partial_{1}}{\longrightarrow} X_{0} \rightarrow 0
$$

in $A$-mod with fixed sequence

$$
\mathbf{d}=\left(\operatorname{dim}_{K} X_{m}, \ldots, \operatorname{dim}_{K} X_{0}\right)=\left(d_{m}, \ldots, d_{0}\right)
$$

of dimensions. Accordingly, we define the variety $\operatorname{Comp}_{\mathbf{d}}^{A}$ to be the following Zariski closed subset of the affine space

$$
\mathbb{A}^{N}=\prod_{0 \leq i \leq m} \operatorname{Hom}_{K}\left(A, \operatorname{End}_{K}\left(K^{d_{i}}\right)\right) \times \prod_{1 \leq i \leq m} \operatorname{Hom}_{K}\left(K^{d_{i}}, K^{d_{i-1}}\right) .
$$

Namely,

$$
\begin{aligned}
\operatorname{Comp}_{\mathbf{d}}^{A}:=\left\{\left(\mathcal{A}^{m}, \ldots, \mathcal{A}^{0}, \partial_{m}, \ldots, \partial_{1}\right) \in \mathbb{A}^{N} \mid \mathcal{A}^{i}=\left(\mathcal{A}_{i j}\right)_{j \leq s} \in \operatorname{Mod}_{d_{i}}^{A},\right. \\
\left.\partial_{i} \mathcal{A}_{i j}=\mathcal{A}_{i-1, j} \partial_{i}, \text { and } \partial_{i-1} \partial_{i}=0 \text { for all } i \leq m \text { and } j \leq s\right\} .
\end{aligned}
$$

We will also label the points of $\operatorname{Comp}_{\mathbf{d}}^{A}$ in the form $\left(\mathcal{A}_{i j}, \partial_{m}, \ldots, \partial_{1}\right)$, as convenience dictates. Clearly, the group $\mathrm{GL}_{\mathbf{d}}=\mathrm{GL}_{d_{m}} \times \cdots \times \mathrm{GL}_{d_{0}}$ acts morphically on Comp $\mathbf{d}_{\mathbf{d}}^{A}$ via

$$
\left(g_{m}, \ldots, g_{0}\right)\left(\mathcal{A}_{i j}, \partial_{m}, \ldots, \partial_{1}\right)=\left(\mathcal{A}_{i j}^{g_{i}}, g_{m-1} \partial_{m} g_{m}^{-1}, \ldots, g_{0} \partial_{1} g_{1}^{-1}\right)
$$


where $\mathcal{A}_{i j}^{g_{i}}$ stands for the conjugate $g_{i} \mathcal{A}_{i j} g_{i}^{-1}$. Moreover, it is straightforward to check the following fact.

Observation 1. The natural map from $\operatorname{Comp}_{\mathbf{d}}^{A}$ to the collection of complexes $\mathbf{X}$ as above, with fixed sequence $\mathbf{d}$ of dimensions, induces a one-to-one correspondence between the orbits of the $\mathrm{GL}_{\mathbf{d}}$-action on $\mathrm{Comp}_{\mathbf{d}}^{A}$ on one hand, and the isomorphism classes of complexes of the described format on the other.

An alternate way of geometrizing complexes is, of course, that of viewing complexes as graded modules over an enlarged algebra; but this approach would be ill-suited to our purpose of relating the geometry of $\operatorname{Comp}_{\mathbf{d}}^{A}$ to the derived category of $A$.

In the following, we will denote points of $\operatorname{Comp}_{\mathbf{d}}^{A}$ by capital letters and the corresponding complexes by bold versions of these letters. Furthermore, we will, from now on, assume that $a_{1}=1, \ldots, a_{s}$ is a $K$-basis of $A$. This provides us with structure constants $c_{j k l} \in K$, arising in the equalities $a_{j} a_{k}=\sum_{l=1}^{s} c_{j k l} a_{l}$. In order to describe the coordinate ring of $\operatorname{Comp}_{\mathbf{d}}^{A}$, we let $X_{i j}$ stand for a $d_{i} \times d_{i}$ matrix of variables representing the entries of the matrix $\mathcal{A}_{i j}$, respectively, and $Y_{i}$ for a $d_{i-1} \times d_{i}$ matrix of variables representing the entries of the map $\partial_{i}$. When we view a sequence $\left(X_{i j}, Y_{m}, \ldots, Y_{1}\right)$ as an element of the coordinate ring of $\operatorname{Comp}_{\mathbf{d}}^{A}$, our setup shows these coordinate matrices to be subject to the following equalities; in fact, $\operatorname{Comp}_{\mathbf{d}}^{A}$ is determined by these requirements:

( $\alpha) X_{i j} X_{i k}=\sum_{l=1}^{s} c_{j k l} X_{i l}$;

(ß) $Y_{i} X_{i j}=X_{i-1, j} Y_{i}$;

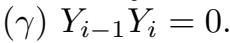

In the upcoming lemma, we will derive a convenient explicit description of the Zariski tangent space $T_{X}\left(\operatorname{Comp}_{\mathbf{d}}^{A}\right)$ of $\operatorname{Comp}_{\mathbf{d}}^{A}$ at a point $X$. Recall that a derivation of a left $A$-module $M$ is a $K$-linear map $\delta: A \rightarrow \operatorname{End}_{K}(M)$ such that $\delta(a b) x=$ $a(\delta(b) x)+\delta(a)(b x)$ for all $a, b \in A$ and $x \in M$.

Lemma 2. Given a point $X=\left(\mathcal{A}^{m}, \ldots, \mathcal{A}^{0}, \partial_{m}, \ldots, \partial_{1}\right) \in \operatorname{Comp}_{\mathbf{d}}^{A}$, the tangent space $T_{X}\left(\mathrm{Comp}_{\mathbf{d}}^{A}\right)$ of $\operatorname{Comp}_{\mathbf{d}}^{A}$ at $X$ consists precisely of those sequences $\left(\delta_{m}, \ldots, \delta_{0}\right.$, $\left.\sigma_{m}, \ldots, \sigma_{1}\right)$ in

$$
\mathbb{A}^{N}=\prod_{0 \leq i \leq m} \operatorname{Hom}_{K}\left(A, \operatorname{End}_{K}\left(K^{d_{i}}\right)\right) \times \prod_{1 \leq i \leq m} \operatorname{Hom}_{K}\left(K^{d_{i}}, K^{d_{i-1}}\right)
$$

which satisfy the following conditions:

(a) Each $\delta_{i}: A \rightarrow \operatorname{End}_{K}\left(K^{d_{i}}\right)$ is a derivation of the left $A$-module $X_{i}$ determined by $\mathcal{A}^{i}=\left(\mathcal{A}_{i j}\right)_{j \leq s}$.

(b) $\sigma_{i} \mathcal{A}_{i j}+\partial_{i} \delta_{i}\left(a_{j}\right)=\mathcal{A}_{i-1, j} \sigma_{i}+\delta_{i-1}\left(a_{j}\right) \partial_{i}$ for all $0 \leq i \leq m$ and $1 \leq j \leq s$; here we identify $\mathcal{A}_{i j}$ with the left multiplication of $X_{i}=\bar{K}^{d_{i}}$ by the element $a_{j}$ of A.

(c) $\sigma_{i-1} \partial_{i}+\partial_{i-1} \sigma_{i}=0$ for all $1 \leq i \leq m$.

Proof. Note that each of the $\delta_{i}$ is determined by the values $\left(\delta_{i}\left(a_{j}\right)\right)_{j \leq s}$. Hence the defining conditions $(\alpha)-(\gamma)$ of $\operatorname{Comp}_{\mathbf{d}}^{A}$ yield the following equations pinning down $T_{X}\left(\operatorname{Comp}_{\mathbf{d}}^{A}\right)$; they are given in the variables $w_{i j}$ representing the maps $\delta_{i}\left(a_{j}\right)$, and $v_{i}$ representing the $K$-endomorphisms $\sigma_{i}: K^{d_{i}} \rightarrow K^{d_{i-1}}$, respectively:

(a) $w_{i j} \mathcal{A}_{i k}+\mathcal{A}_{i j} w_{i k}=\sum_{l=0}^{s} c_{j k l} w_{i l}$,

(b) $v_{i} \mathcal{A}_{i j}+\partial_{i} w_{i j}=w_{i-1, j} \partial_{i}+\mathcal{A}_{i-1, j} v_{i}$, 
(c) $v_{i-1} \partial_{i}+\partial_{i-1} v_{i}=0$,

for all eligible indices $i, j, k$. But these conditions are clearly tantamount to the ones listed in our claim.

Next we follow the traditional road of mapping the tangent space of a module variety at a point $x$ to the group of self-extensions of the module represented by $x$. Namely, we fix $X \in \mathrm{Comp}_{\mathbf{d}}^{A}$, and assign to any point $\left(\delta_{m}, \ldots, \delta_{0}, \sigma_{m}, \ldots, \sigma_{1}\right)$ in the tangent space $T_{X}\left(\mathrm{Comp}_{\mathbf{d}}^{A}\right)$ a short exact sequence $0 \rightarrow \mathbf{X} \rightarrow \mathbf{Z} \rightarrow \mathbf{X} \rightarrow 0$ of complexes as follows.

Remark 3. Let $X \in \mathrm{Comp}_{\mathbf{d}}^{A}$ as before.

(1) Condition (a) of Lemma 2 implies that the following is a left $A$-module structure on $Z_{i}=K^{d_{i}} \oplus K^{d_{i}}$ : Namely, we define left multiplication of $Z_{i}$ by $a \in A$ as left multiplication by $\left(\begin{array}{cc}\mathcal{A}^{i}(a) & \delta_{i}(a) \\ 0 & \mathcal{A}^{i}(a)\end{array}\right)$, viewing the elements $Z_{i}$ as column vectors.

(2) Condition (c) of Lemma 2 tells us that we can supplement the $Z_{i}$ to a chain complex $\mathbf{Z}$ of $K$-vector spaces by introducing the differentials $\partial_{i}^{\mathbf{Z}}=\left(\begin{array}{cc}\partial_{i} & \sigma_{i} \\ 0 & \partial_{i}\end{array}\right)$.

(3) Condition (b) of Lemma 2, finally, shows that the complex $\mathbf{Z}$ just defined is, in fact, a complex of $A$-modules.

(4) Let $0 \rightarrow X_{i} \rightarrow Z_{i} \rightarrow X_{i} \rightarrow 0$ be the canonical sequences, given by injection of $X_{i}$ into the first component of $Z_{i}$, followed by projection onto the second component. These short exact sequences in $A$-mod are compatible with the differentials, thus yielding a short exact sequence $0 \rightarrow \mathbf{X} \rightarrow \mathbf{Z} \rightarrow \mathbf{X} \rightarrow 0$ of chain complexes.

Now suppose that $\mathbf{X}, \mathbf{Y}$ are chain complexes which are bounded above, but not necessarily finite. We will consider the group $\operatorname{Ext}^{1}(\mathbf{X}, \mathbf{Y})$ of (equivalence classes) of extensions $0 \rightarrow \mathbf{Y} \rightarrow \mathbf{E} \rightarrow \mathbf{X} \rightarrow 0$ in the category of complexes of $A$-modules. In analogy with Ext-groups of $A$-modules, $\operatorname{Ext}^{1}(\mathbf{X}, \mathbf{Y})$ actually carries a $K$-vector space structure.

For any point $X \in \mathrm{Comp}_{\mathbf{d}}^{A}$, Remark 3 provides us with a $K$-linear map

$$
\chi: T_{X}\left(\operatorname{Comp}_{\mathbf{d}}^{A}\right) \rightarrow \operatorname{Ext}^{1}(\mathbf{X}, \mathbf{X}),
$$

sending an element of $T_{X}\left(\operatorname{Comp}_{\mathbf{d}}^{A}\right)$ to the class of the self-extension $0 \rightarrow \mathbf{X} \rightarrow \mathbf{Z} \rightarrow$ $\mathbf{X} \rightarrow 0$ of $\mathbf{X}$ described above. The proof of the following proposition is quite similar to that of Voigt's Lemma ([19, Chap. 2, Section 3.4]; see also [5, Theorem 1.6]). Since Voigt's original argument (couched in German) carries over smoothly to our setting, we include only a brief outline (for the sake of the reader not familiar with German). Recall that an extension $0 \rightarrow \mathbf{Y} \rightarrow \mathbf{E} \rightarrow \mathbf{X} \rightarrow 0$ is semisplit in the sense of Verdier [18, pp. 272-273] if, in each degree $i$, the pertinent short exact sequence of $A$-modules splits.

Proposition 4. Suppose that $K$ is an algebraically closed field. Then, given any $X \in \mathrm{Comp}_{\mathbf{d}}^{A}$, the kernel of the map $\chi$ equals the tangent space of $G . X$ at $X$. In other words, $\chi$ induces a vector space embedding

$$
T_{X}\left(\operatorname{Comp}_{\mathbf{d}}^{A}\right) / T_{X}(G . X) \rightarrow \operatorname{Ext}^{1}(\mathbf{X}, \mathbf{X}) .
$$

Moreover, all semisplit self-extensions of $\mathbf{X}$ belong to the image of $\chi$.

Proof. The canonical morphism $G \rightarrow G$.X is separable (since the stabilizer subgroup of $X$ in $G$ arises as the solution set of a system of linear equations), i.e., this map has a surjective differential

$$
T_{e}(G) \rightarrow T_{X}(G . X) \subseteq T_{X}\left(\operatorname{Comp}_{\mathbf{d}}^{A}\right) .
$$


In the description of the tangent space $T_{X}\left(\mathrm{Comp}_{\mathbf{d}}^{A}\right)$ given in Lemma 2, this identifies $T_{X}(G . X)$ as being the following subset of $T_{X}\left(\operatorname{Comp}_{\mathbf{d}}^{A}\right)$ : Namely, the set of those elements $\left(\delta_{m}, \ldots, \delta_{0}, \sigma_{m}, \ldots, \sigma_{1}\right)$ in $T_{X}\left(\operatorname{Comp}_{\mathbf{d}}^{A}\right)$ for which there is a sequence $t=$ $\left(t_{m}, \ldots, t_{0}\right) \in \prod_{i=0}^{m} \operatorname{End}_{K}\left(K^{d_{i}}\right)$ such that the following equalities hold for all eligible $i$ and $j$ :

- $\delta_{i}\left(a_{j}\right)=t_{i} \mathcal{A}_{i j}-\mathcal{A}_{i j} t_{i}$, and

- $\sigma_{i}=t_{i-1} \partial_{i}-\partial_{i} t_{i}$.

(These equalities can be gleaned directly from Voigt's argument for modules by identifying the complex $\mathbf{X}$ with a graded module $\bigoplus_{i=0}^{m} X_{i}$ over the finite dimensional algebra $A\left[\partial_{1}, \ldots, \partial_{m}\right] /\left(\partial_{1}^{2}, \ldots, \partial_{m}^{2}\right)$; here the operation of $A$ on the direct sum is extended via $\partial_{i} \cdot\left(x_{m}, \ldots, x_{0}\right)=\partial_{i}\left(x_{i}\right) \in X_{i-1}$ for $1 \leq i \leq m$.)

It is readily verified that the described subset of $T_{X}\left(\mathrm{Comp}_{\mathbf{d}}^{A}\right)$ coincides with the kernel of $\chi$. Indeed, splitness of the extension $0 \rightarrow \mathbf{X} \rightarrow \mathbf{Z} \rightarrow \mathbf{X} \rightarrow 0$ of complexes coming with the point $\left(\delta_{m}, \ldots, \delta_{0}, \sigma_{m}, \ldots, \sigma_{1}\right)$ of $T_{X}\left(\mathrm{Comp}_{\mathbf{d}}^{A}\right)$ is equivalent to the existence of a section for the epimorphism $\mathbf{Z} \rightarrow \mathbf{X}$. Since the latter map just projects the $Z_{i}=X_{i} \oplus X_{i}$ onto the second components, such sections are precisely the chain maps $\mathbf{X} \rightarrow \mathbf{Z}$ of the form $t^{\prime}=\left(t_{i}, 1_{X_{i}}\right)_{0 \leq i \leq m}$, where the family $\left(t_{i}\right)$ belongs to $\prod_{i=0}^{m} \operatorname{End}_{A}\left(X_{i}\right)$. But the condition that $t_{i}^{\prime}: X_{i} \rightarrow Z_{i}$ be an $A$-module homomorphism is clearly tantamount to the requirement that $t_{i} \mathcal{A}_{i j}=\mathcal{A}_{i j} t_{i}+\delta_{i}\left(a_{j}\right)$, while the stipulation that a family $\left(t_{i}\right) \in \prod_{i=0}^{m} \operatorname{Hom}_{K}\left(K^{d_{i}}\right)$ be compatible with the differentials of $\mathbf{X}$ and $\mathbf{Z}$ amounts to the equality $t_{i-1} \partial_{i}=\partial_{i} t_{i}+\sigma_{i}$. This completes the proof of the fact that $T_{X}(G . X)$ coincides with the kernel of $\chi$.

For the final comment, note that the differential of any semisplit self-extension $0 \rightarrow \mathbf{X} \rightarrow \mathbf{Z} \rightarrow \mathbf{X} \rightarrow 0$ of $\mathbf{X}$, with splitting $Z_{i}=X_{i} \oplus X_{i}$ in degree $i$, has the form $\partial_{i}^{\mathbf{Z}}=\left(\begin{array}{cc}\partial_{i} & \sigma_{i} \\ 0 & \partial_{i}\end{array}\right)$ relative to such a splitting, where $\partial_{i}$ is the $i$-th differential of $\mathbf{X}$ and $\sigma_{i} \in \operatorname{Hom}_{A}\left(X_{i}, X_{i-1}\right)$ satisfies condition (c) of Lemma 2. Consequently, Remark 3 shows that the semisplit self-extensions of $\mathbf{X}$ are precisely the images under $\chi$ of the vectors $\left(0, \ldots, 0, \sigma_{m}, \ldots, \sigma_{1}\right)$ in the tangent space $T_{X}\left(\operatorname{Comp}_{\mathbf{d}}^{A}\right)$.

Following Verdier's conventions in [18, we denote the category of right bounded complexes of finite dimensional left $A$-modules by $C^{-}(A$-mod $)$, its quotient category modulo homotopy by $K^{-}(A$-mod), and the corresponding derived category by $D^{-}(A$-mod $)$. Analogously, $C^{b}(A$-mod $), K^{b}(A$-mod $)$, and $D^{b}(A$-mod $)$ stand for the category of bounded complexes, for the quotient category modulo homotopy, and the pertinent derived category, respectively. According to [18, pp. 294-295], every element $0 \rightarrow \mathbf{Y} \rightarrow \mathbf{E} \rightarrow \mathbf{X} \rightarrow 0$ of $\operatorname{Ext}^{1}(\mathbf{X}, \mathbf{Y})$ gives rise to a distinguished triangle $\mathbf{Y} \rightarrow \mathbf{E} \rightarrow \mathbf{X} \rightarrow \mathbf{Y}[1]$ in $D^{-}(A$-mod). Here $\mathbf{Y}[1]$ denotes the shifted complex which carries $Y_{n-1}$ in the slot labeled $n$. This provides us with a $K$-linear map $\xi: \operatorname{Ext}^{1}(\mathbf{X}, \mathbf{Y}) \longrightarrow \operatorname{Hom}_{D^{-}(A \text {-mod })}(\mathbf{X}, \mathbf{Y}[1])$ which assigns to an extension as above the connecting morphism $\mathbf{X} \rightarrow \mathbf{Y}[1]$ of the corresponding triangle. Our next intermediate goal is to scrutinize this map for complexes $\mathbf{X}$ which are 'close' to being projective.

Definition. A complex $\mathbf{X}: \cdots \rightarrow X_{i+1} \rightarrow X_{i} \rightarrow \cdots$ in $C^{-}(A$-mod $)$ is called projective if all of the terms $X_{i}$ are projective $A$-modules.

Now suppose that $\mathbf{X} \in C^{b}(A$-mod). If $\mathbf{X}$ is nonzero, then the largest integer $m$ with $X_{m} \neq 0$ will be referred to as the left degree of $\mathbf{X}$. We will call $\mathbf{X}$ almost projective if either $\mathbf{X}$ is projective or else $X_{m}$ is the only non-projective term of the complex. 
It is clear that, under the natural map $C^{b}(A$-mod $) \rightarrow D^{b}(A$-mod $)$, every object in the derived category is represented by an almost projective complex.

One of the assets of almost projective complexes is a convenient lifting property for maps in the derived category: If $\mathbf{X}$ and $\mathbf{Y}$ are both almost projective with coinciding left degree, then any map in $\operatorname{Hom}_{D^{b}(A \text {-mod })}(\mathbf{X}, \mathbf{Y})$ lifts to a chain map in $\operatorname{Hom}_{C^{b}(A \text {-mod })}(\mathbf{X}, \mathbf{Y})$ : Indeed, we may restrict our attention to bounded almost projective complexes for that purpose. So suppose that $\mathbf{X}$ is of the form

$$
0 \rightarrow X_{m} \stackrel{\partial_{m}}{\longrightarrow} X_{m-1} \stackrel{\partial_{m-1}}{\longrightarrow} \ldots \stackrel{\partial_{1}}{\longrightarrow} X_{0} \rightarrow 0,
$$

where all of the left $A$-modules $X_{i}$, except for $X_{m}$ possibly, are projective; write $\mathbf{Y}$ in the same format, with terms $Y_{i}$. If we let $\cdots \rightarrow P_{m+1} \stackrel{p_{m+1}}{\longrightarrow} P_{m} \stackrel{p_{m}}{\longrightarrow} X_{m} \rightarrow 0$ be a projective resolution of $X_{m}$, the image of $\mathbf{X}$ in $D^{-}(A$-mod) clearly coincides with that of the projective complex $\mathbf{X}^{\prime}$, given by

$$
\ldots P_{m+1} \stackrel{p_{m+1}}{\longrightarrow} P_{m} \stackrel{\partial_{m} p_{m}}{\longrightarrow} X_{m-1} \stackrel{\partial_{m-1}}{\longrightarrow} \ldots \stackrel{\partial_{1}}{\longrightarrow} X_{0} \rightarrow 0 .
$$

Analogously, define a projective complex $\mathbf{Y}^{\prime}$ based on $\mathbf{Y}$. Now any homomorhism in $\operatorname{Hom}_{D^{-}(A \text {-mod })}\left(\mathbf{X}^{\prime}, \mathbf{Y}^{\prime}\right)$ is induced by a chain map

$$
\eta \in \operatorname{Hom}_{C^{-}(A \text {-mod })}\left(\mathbf{X}^{\prime}, \mathbf{Y}^{\prime}\right) .
$$

But $p_{m}$ is the cokernel of $p_{m+1}$, and therefore $\eta$ induces a chain map in

$$
\operatorname{Hom}_{C^{b}(A \text {-mod })}(\mathbf{X}, \mathbf{Y})
$$

having the same image as $\eta$ in $D^{b}(A$-mod).

Again suppose that $\mathbf{X} \in C^{b}(A$-mod $)$ is almost projective. Along the line suggested by the previous paragraph, we associate another almost projective complex $\mathbf{X}^{(1)}$ to $\mathbf{X}$, by potentially extending $\mathbf{X}$ to the left as follows: Set $\mathbf{X}^{(1)}=\mathbf{X}$ if $\mathbf{X}$ is projective. Otherwise, we denote the left degree of $\mathbf{X}$ by $m$, let $p: X_{m}^{(1)} \rightarrow X_{m}$ be a projective cover of $X_{m}$ with kernel $\partial_{m+1}^{\mathbf{X}^{(1)}}: X_{m+1}^{(1)} \rightarrow X_{m}^{(1)}$, and define $\mathbf{X}^{(1)}$ to be the complex

$$
0 \rightarrow X_{m+1}^{(1)} \rightarrow X_{m}^{(1)} \rightarrow X_{m-1} \rightarrow \cdots \rightarrow X_{0} \rightarrow 0
$$

where the $m$-th differential of the complex $\mathbf{X}^{(1)}$ is $\partial_{m}^{\mathbf{X}^{(1)}}=\partial_{m}^{\mathbf{X}} p$. Clearly, $\mathbf{X}^{(1)}$ comes with a natural map $f: \mathbf{X}^{(1)} \rightarrow \mathbf{X}$ of chain complexes, given by $f_{m}=p$, $f_{i}=0$ for $i \geq m+1$, and $f_{i}=\operatorname{id}_{X_{i}}$ for $i \leq m-1$. By setting $\mathbf{X}^{(0)}=\mathbf{X}$ and continuing inductively via $\mathbf{X}^{(r+1)}=\left(\mathbf{X}^{(r)}\right)^{(1)}$, we thus obtain a sequence

$$
\cdots \rightarrow \mathbf{X}^{(r)} \rightarrow \mathbf{X}^{(r-1)} \rightarrow \cdots \rightarrow \mathbf{X}^{(1)} \rightarrow \mathbf{X}^{(0)}
$$

of chain maps in $C^{-}(A$-mod $)$, which, in turn, induces a sequence

$$
\operatorname{Ext}^{1}(\mathbf{X}, \mathbf{Y}) \rightarrow \operatorname{Ext}^{1}\left(\mathbf{X}^{(1)}, \mathbf{Y}\right) \rightarrow \operatorname{Ext}^{1}\left(\mathbf{X}^{(2)}, \mathbf{Y}\right) \rightarrow \cdots
$$

of $K$-linear maps for each $\mathbf{Y} \in C^{-}\left(A\right.$-mod). Note that Verdier's map $\xi: \operatorname{Ext}^{1}(\mathbf{X}, \mathbf{Y})$ $\rightarrow \operatorname{Hom}_{D^{-}(A \text {-mod })}(\mathbf{X}, \mathbf{Y}[1])$ factors through these vector space homomorphisms, since all of the canonical maps $\mathbf{X}^{(r)} \rightarrow \mathbf{X}^{(r-1)}$ become isomorphisms in the homotopy category $K^{-}(A$-mod). 
We begin with an auxiliary point which sometimes allows us to replace $\mathbf{X}$ by $\mathbf{X}^{(1)}$ in the first argument of Ext ${ }^{1}$ without penalty.

Lemma 5. Let $\mathbf{X}, \mathbf{Y} \in C^{b}(A$-mod) be nonzero almost projective complexes whose left degrees coincide. Then the canonical K-linear map

$$
\operatorname{Ext}^{1}(\mathbf{X}, \mathbf{Y}) \rightarrow \operatorname{Ext}^{1}\left(\mathbf{X}^{(1)}, \mathbf{Y}\right)
$$

is bijective.

Proof. The coinciding left degree of $\mathbf{X}$ and $\mathbf{Y}$ is denoted by $m$. Moreover, we write $\mathbf{Z}$ for $\mathbf{X}^{(1)}$, and $f$ for the canonical chain map $\mathbf{Z} \rightarrow \mathbf{X}$; this means, in particular, that $f_{m}: Z_{m} \rightarrow X_{m}$ is a projective cover, and $f_{i}=\operatorname{id}_{X_{i}}$ for $i \leq m-1$. The $K$-linear map addressed in our claim, finally, is denoted by $h: \operatorname{Ext}^{1}(\mathbf{X}, \mathbf{Y}) \rightarrow \operatorname{Ext}^{1}(\mathbf{Z}, \mathbf{Y})$.

We start by proving surjectivity of $h$. Suppose

$$
\phi: 0 \rightarrow \mathbf{Y} \stackrel{\rho}{\rightarrow} \mathbf{F} \stackrel{\sigma}{\rightarrow} \mathbf{Z} \rightarrow 0
$$

represents a class in $\operatorname{Ext}^{1}(\mathbf{Z}, \mathbf{Y})$. To construct a preimage

$$
\epsilon: 0 \rightarrow \mathbf{Y} \stackrel{\mu}{\longrightarrow} \mathbf{E} \stackrel{\nu}{\rightarrow} \mathbf{X} \rightarrow 0
$$

of (the class of) $\phi$ under $h$, we start by setting $E_{i}=0$ for $i \geq m+1$ and $E_{i}=F_{i}$ for $i \leq m-1$; the pertinent components of the differential $\partial^{\mathbf{E}}$ and those of the chain maps $\mu, \nu$ are as follows: $\partial_{i}^{\mathbf{E}}=\partial_{i}^{\mathbf{F}}, \mu_{i}=\rho_{i}$, and $\nu_{i}=\sigma_{i}$ for $i \leq m-1$, the definitions to the left of the $m$-th position being obvious. The $A$-module $E_{m}$ and the corresponding differential of $\mathbf{E}$ are defined by the requirements that $g_{m}: F_{m} \rightarrow E_{m}$ be the cokernel of $\partial_{m+1}^{\mathbf{F}}$, and $\partial_{m}^{\mathbf{E}}$ be the unique map in $\operatorname{Hom}_{A}\left(E_{m}, F_{m-1}\right)$ such that $\partial_{m}^{\mathbf{F}}=\partial_{m}^{\mathbf{E}} g_{m}$. Moreover, set $\mu_{m}=g_{m} \rho_{m}$, and let $\nu_{m}: E_{m} \rightarrow X_{m}$ be the unique homomorphism with $f_{m} \sigma_{m}=\nu_{m} g_{m}$; the map $\nu_{m}$ is, once more, furnished by the universal property of $g_{m}$. Clearly, $\mu: \mathbf{X} \rightarrow \mathbf{E}$ is a chain map. The same is true for $\nu: \mathbf{E} \rightarrow \mathbf{X}$; indeed, a straightforward diagram chase yields $\partial_{m}^{\mathbf{X}} \nu_{m}=\nu_{m-1} \partial_{m}^{\mathbf{E}}$. It is now routine to check that $\epsilon$ yields a preimage of $\phi$ under $h$.

To verify injectivity of $h$, suppose that the class of $\epsilon: 0 \rightarrow \mathbf{Y} \stackrel{\mu}{\rightarrow} \mathbf{E} \stackrel{\nu}{\rightarrow} \mathbf{X} \rightarrow 0$ is mapped to zero by $h$. This means that the upper row of the following pullback diagram of complexes splits:

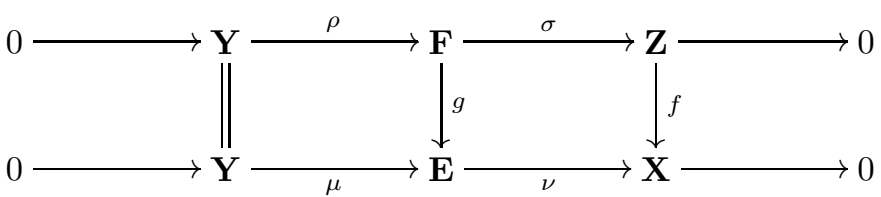

In order to show that $\epsilon$ is trivial, i.e., that the lower row splits as well, let $\tau=\left(\tau_{n}\right)_{n \in \mathbb{N}}$ be a section for $\sigma$. To construct a section $\pi$ for $\nu$, we start by setting $\pi_{i}=0$ for $i \geq m+1$, and $\pi_{i}=g_{i} \tau_{i}$ for $i \leq m-1$. This setup is recorded in the 
following diagram:

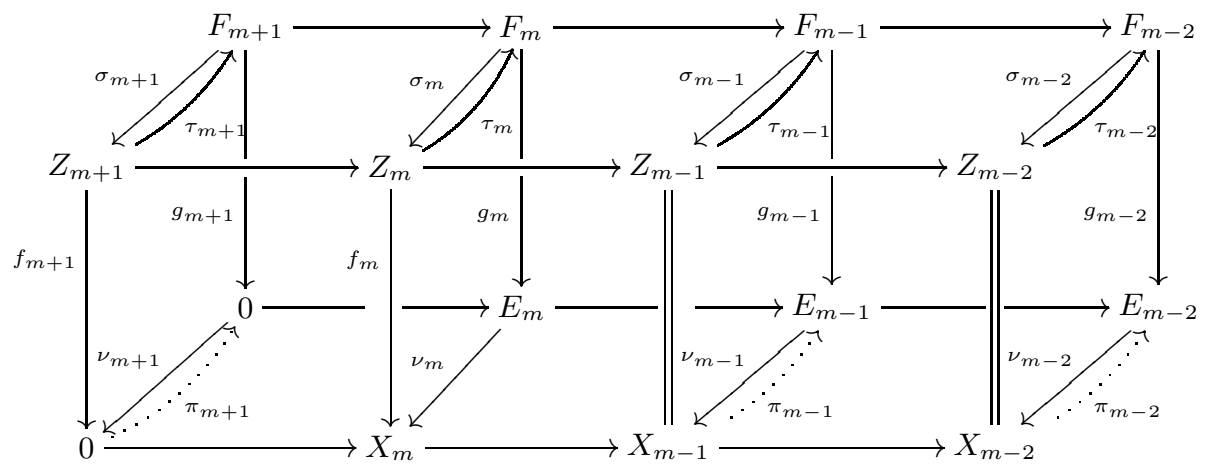

Note that all of the squares in this diagram commute, so that our task is reduced to finding a section $\pi_{m}$ for $\nu_{m}$ such that the two bottom squares adjacent to $\pi_{m}$ commute. This is automatic for the left-hand square, irrespective of our choice of $\pi_{m}$. To construct $\pi_{m}$ with the property that $\partial_{m}^{\mathbf{E}} \pi_{m}=\pi_{m-1} \partial_{m}^{\mathbf{X}}$, we recall that $f_{m}$ is the cokernel of the differential $\partial_{m+1}^{\mathbf{Z}}$. This provides us with a unique homomorphism $\pi_{m}: X_{m} \rightarrow E_{m}$ having the property that $g_{m} \tau_{m}=\pi_{m} f_{m}$, for $g_{m} \tau_{m} \partial_{m+1}^{\mathbf{Z}} \sigma_{m+1}=\partial_{m+1}^{\mathbf{E}} g_{m+1}=0$ yields $g_{m} \tau_{m} \partial_{m+1}^{\mathbf{Z}}=0$. It is now routine to check that $\pi=\left(\pi_{i}\right)_{i \in \mathbb{Z}}$ is a section for $\nu$, as required. This completes the argument.

Modules $M, N \in A$-mod can of course be viewed as stalk complexes $\mathbf{M}, \mathbf{N}$ concentrated in degree 0 . In this situation, Verdier's map provides us with isomorphisms

$$
\operatorname{Ext}^{1}(\mathbf{M}, \mathbf{N}) \cong \operatorname{Hom}_{D^{-}(A-\bmod )}(\mathbf{M}, \mathbf{N}[1]) .
$$

The following lemma shows that the good behavior of Verdier's map extends, at least partially, to more general complexes, which provides the missing link towards the main result of this section.

Lemma 6. Let $\mathbf{X}, \mathbf{Y}$ be nonzero complexes in $C^{-}(A$-mod $)$. Then Verdier's map

$$
\xi: \operatorname{Ext}^{1}(\mathbf{X}, \mathbf{Y}) \longrightarrow \operatorname{Hom}_{D^{-}}(A \text {-mod })(\mathbf{X}, \mathbf{Y}[1])
$$

is injective if either

(1) $\mathbf{X}$ is a projective complex, or else

(2) $\mathbf{X}$ and $\mathbf{Y}$ are bounded almost projective complexes such that the left degree of $\mathbf{X}$ is larger than or equal to the left degree of $\mathbf{Y}$.

Moreover, if $\mathbf{X}$ is as in (1) or (2) and $\mathbf{Y}$ is projective, then $\xi$ is bijective.

Proof. We show injectivity of $\xi$ simultaneously for (1) and (2). In case (2), we denote by $k$ the left degree of $\mathbf{X}$, by $m$ that of $\mathbf{Y}$, and observe that Lemma 5 permits us to derive the claim for $k=m$ from that for $k=m+1$. In other words, we may, w.l.o.g., assume that either both $\mathbf{X}$ and $\mathbf{Y}$ are projective or both are bounded almost projective with $k \geq m+1$. Note that, in this situation, any extension $\epsilon: 0 \rightarrow \mathbf{Y} \stackrel{\mu}{\longrightarrow} \mathbf{E} \stackrel{\nu}{\rightarrow} \mathbf{X} \rightarrow 0$ has the property that $0 \rightarrow Y_{i} \stackrel{\mu_{i}}{\longrightarrow} E_{i} \stackrel{\nu_{i}}{\longrightarrow} X_{i} \rightarrow 0$ splits (i.e., the extension $\epsilon$ is semisplit in the sense of [18] pp. 272-273]). For each $i \in \mathbb{Z}$, let $\pi_{i}^{\prime}$ be a section of $\nu_{i}$. We will see that the assumption ' $\xi(\epsilon)=0$ ' allows us to adjust the family $\left(\pi_{i}^{\prime}\right)$ so as to furnish a section for $\nu$. To that end, suppose that $\xi(\epsilon)$ is represented by a chain map $\phi: \mathbf{X} \rightarrow \mathbf{Y}[1]$. Let $\mathbf{P} \rightarrow \mathbf{X}$ be the projective resolution 
of $\mathbf{X}$ in $K^{-}(A$-mod) and $\tilde{\phi}: \mathbf{P} \rightarrow \mathbf{Y}[1]$ its composition with $\phi$; then the morphism $\tilde{\phi}$ in $K^{-}(A$-mod $)$ also represents $\xi(\epsilon)$ in $D^{-}(A$-mod $)$, in view of the canonical isomorphism $\operatorname{Hom}_{K^{-}(A \text {-mod })}(\mathbf{X}, \mathbf{Y}[1]) \cong \operatorname{Hom}_{K^{-}(A \text {-mod })}(\mathbf{P}, \mathbf{Y}[1])$ (see [18, p. 299]). We further observe that $\xi(\epsilon)=0$ if and only if $\tilde{\phi}$ is trivial in $K^{-}(A$-mod), for the quotient map $\operatorname{Hom}_{K^{-}(A \text {-mod })}(\mathbf{P}, \mathbf{Y}[1]) \rightarrow \operatorname{Hom}_{D^{-}(A \text {-mod })}(\mathbf{P}, \mathbf{Y}[1])$ is injective. Our hypothesis on the left degrees of $\mathbf{X}$ and $\mathbf{Y}$ guarantees that $\tilde{\phi}$ is nullhomotopic precisely when $\phi$ is nullhomotopic. Consequently, the assumption that $\xi(\epsilon)$ is zero supplies us with a homotopy $\psi$ from $\phi$ to zero. We deduce that $\pi=\left(\pi_{n}^{\prime}-\mu_{n} \psi_{n}\right)_{n \in \mathbb{Z}}$ is a chain map $\mathbf{X} \rightarrow \mathbf{E}$ such that $\nu \pi=1_{\mathbf{X}}$, which shows that $\epsilon$ is indeed trivial.

Now suppose that $\mathbf{Y}$ is projective. In that case, we have isomorphisms

$$
\operatorname{Hom}_{D^{-}(A-\bmod )}(\mathbf{X}, \mathbf{Y}[1]) \cong \operatorname{Hom}_{K^{-}(A-\bmod )}(\mathbf{P}, \mathbf{Y}[1]) \cong \operatorname{Hom}_{K^{-}(A-\bmod )}(\mathbf{X}, \mathbf{Y}[1])
$$

where $\mathbf{P}$ is a projective cover of $\mathbf{X}$. Hence all we need to check is that the homotopy class of any chain map $\psi: \mathbf{X} \rightarrow \mathbf{Y}[1]$ occurs in the image of the map $\operatorname{Ext}^{1}(\mathbf{X}, \mathbf{Y}) \rightarrow$ $\operatorname{Hom}_{K^{-}(A \text {-mod })}(\mathbf{X}, \mathbf{Y}[1])$ induced by $\xi$. But if $\mathbf{C}$ is the mapping cone of $\psi$, then clearly the class of the extension $0 \rightarrow \mathbf{Y} \rightarrow \mathbf{C}[-1] \rightarrow \mathbf{X} \rightarrow 0$ in $\operatorname{Ext}^{1}(\mathbf{X}, \mathbf{Y})$ is such a preimage of $\psi$.

From the preceding lemmas it is now easy to glean

Theorem 7. Suppose that $K$ is algebraically closed, and let $\mathbf{X}: 0 \rightarrow X_{m} \rightarrow \cdots \rightarrow$ $X_{0} \rightarrow 0$ be an almost projective complex of finite dimensional A-modules, represented by a point $X$ in $\operatorname{Comp}_{\mathbf{d}}^{A}$, where $\mathbf{d}=\left(\operatorname{dim} X_{m}, \ldots, \operatorname{dim} X_{0}\right)$. Moreover, let $T_{X}\left(\operatorname{Comp}_{\mathbf{d}}^{A}\right)$ and $T_{X}(G . X)$ be the tangent spaces at $X$ of $\operatorname{Comp}_{\mathbf{d}}^{A}$ and $G . X$, respectively. Then there exists a canonical $K$-linear embedding

$$
T_{X}\left(\operatorname{Comp}_{\mathbf{d}}^{A}\right) / T_{X}(G \cdot X) \longrightarrow \operatorname{Hom}_{D^{b}(A-\bmod )}(\mathbf{X}, \mathbf{X}[1]) .
$$

In case $\mathbf{X}$ is a projective complex, this embedding is an isomorphism.

Proof. We compose the vector space homomorphism

$$
\chi: T_{X}\left(\operatorname{Comp}_{\mathbf{d}}^{A}\right) \rightarrow \operatorname{Ext}^{1}(\mathbf{X}, \mathbf{X})
$$

introduced after Remark 3 with Verdier's map

$$
\xi: \operatorname{Ext}^{1}(\mathbf{X}, \mathbf{X}) \rightarrow \operatorname{Hom}_{D^{-}(A-\bmod )}(\mathbf{X}, \mathbf{X}[1])
$$

to obtain a vector space homomorphism

$$
\eta: T_{X}\left(\operatorname{Comp}_{\mathbf{d}}^{A}\right) \rightarrow \operatorname{Hom}_{D^{-}(A-\bmod )}(\mathbf{X}, \mathbf{X}[1]) .
$$

Observe that the kernel of $\eta$ coincides with $T_{X}(G . X)$, since $\operatorname{Ker}(\chi)=T_{X}(G . X)$ by Proposition 4 , while $\xi$ is an injection by Lemma 6 . Consequently, $\eta$ induces an embedding of $T_{X}\left(\operatorname{Comp}_{\mathbf{d}}^{A}\right) / T_{X}(G . X)$ into $\operatorname{Hom}_{D^{-}(A-\bmod )}(\mathbf{X}, \mathbf{X}[1])$, as required.

For the final claim, let $\mathbf{X}$ be projective. Then $\xi \chi$ is surjective, since $\chi$ is surjective by the last part of Proposition 4 , and $\xi$ is surjective by the last statement of Lemma 6.

As in the case of the variety $\operatorname{Mod}_{d}^{A}$ of $d$-dimensional $A$-modules, Theorem 7 entails a number of interesting consequences. We just point out two of them, the second of which we will need in the sequel. Since the proofs are analogous to those for the module-theoretic counterparts (see [5]), we leave them to the reader. 
Corollary 8. Suppose that $K$ is algebraically closed and $\mathbf{X} \in C^{b}(A$-mod) a bounded almost projective complex such that $\operatorname{Hom}_{D^{b}(A \text {-mod })}(\mathbf{X}, \mathbf{X}[1])=0$. If $X \in \mathrm{Comp}_{\mathbf{d}}^{A}$ is a point representing $\mathbf{X}$, then the $G$-orbit of $X$ is open in $\operatorname{Comp}_{\mathbf{d}}^{A}$.

Corollary 9. Again suppose that $K$ is algebraically closed. Given any finite sequence $\mathbf{d}=\left(d_{m}, \ldots, d_{0}\right)$ of natural numbers, there are - up to isomorphism in $C^{b}(A$-mod $)$ - only finitely many almost projective complexes $\mathbf{X}$ of A-modules satisfying the following two conditions:

(1) $\operatorname{Hom}_{D^{b}(A \text {-mod })}(\mathbf{X}, \mathbf{X}[1])=0$, and

(2) $\operatorname{dim}_{K} X_{n}=d_{n}$, for all $n \in \mathbb{Z}$.

\section{Faithfully Balanced tWo-Sided COMPLEXeS AND INVARIANCE OF $\operatorname{Out}(A)^{0}$}

Several of the ideas underlying this section have precursors which were developed in a paper by Guil-Asensio and the second-named author [7]. Major portions of our auxiliary results can be readily generalized to algebras over commutative rings; in particular, this is true for Proposition 10 below. To facilitate the reading with a unified blanket hypothesis, we will, however, continue to assume that $A$ is a finite dimensional algebra over an algebraically closed field $K$.

Of course, we can view a chain complex $\mathbf{X} \in C(A$-Mod) as a pair $(\mathbf{X}, \lambda)$, where $\mathbf{X} \in C(K$-Mod $)$ and $\lambda: A \rightarrow \operatorname{End}_{C(K \text {-Mod })}(\mathbf{X})$ is a $K$-algebra homomorphism. Accordingly, given another finite dimensional $K$-algebra $B$, an $A$ - $B$-bimodule complex ${ }_{A} \mathbf{X}_{B}$ amounts to a chain complex $\mathbf{X}$ of $K$-spaces, combined with an algebra homomorphism $\tau: A \otimes_{K} B^{\mathrm{op}} \rightarrow \operatorname{End}_{C(K \text {-Mod })}(\mathbf{X})$. We will call a bimodule complex ${ }_{A} \mathbf{X}_{B}$ almost projective if both ${ }_{A} \mathbf{X}$ and $\mathbf{X}_{B}$ are almost projective in the sense of Section 2; recall that, in particular, this means that $\mathbf{X}$ is a right bounded complex of finite dimensional modules. By adapting standard terminology for modules to this context (see, e.g., 1]), we moreover define:

Definition. A bimodule complex ${ }_{A} \mathbf{X}_{B}$ will be called faithfully balanced provided that both of the canonical $K$-algebra homomorphisms

$$
\lambda=\tau(-, 1): A \rightarrow \operatorname{End}_{C(\operatorname{Mod}-B)}\left(\mathbf{X}_{B}\right), \quad \rho=\tau(1,-): B \rightarrow \operatorname{End}_{C(A-\operatorname{Mod})}\left({ }_{A} \mathbf{X}\right)^{\mathrm{op}}
$$

are isomorphisms.

Whenever we let endomorphisms of a complex $\mathbf{Y}$ of left $A$-modules act on the right of $\mathbf{Y}$, we record this by referring to them as maps in $\operatorname{End}_{C(A \text {-mod })}(\mathbf{Y})^{\text {op }}$. The existence of a faithfully balanced bimodule complex ${ }_{A} \mathbf{X}_{B}$ entails a fairly tight connection between the algebras $A$ and $B$, as the following two results suggest.

Proposition 10. Whenever there exists a faithfully balanced bimodule complex ${ }_{A} \mathbf{X}_{B}$, the centers of $A$ and $B$ are isomorphic.

Proof. Set $E=\operatorname{End}_{C(K \text {-Mod })}(\mathbf{X})$. The canonical embeddings of algebras $\lambda: A \rightarrow E$ and $\rho: B^{\mathrm{op}} \rightarrow E$ take the centers of $\mathrm{A}$ and $\mathrm{B}$ to the same subalgebra $E^{\prime}$ of $E$, namely to $E^{\prime}=\operatorname{End}_{C(A-M o d-B)}(\mathbf{X})$.

As in Section 1, we denote by $\operatorname{Out}(A)$ the group of outer automorphisms of $A$, and by $\operatorname{Out}(A)^{0}$ its identity component. It is well-known (cf. [6], Theorem 1) that $\operatorname{Out}(A)$ embeds naturally into the Picard group Pic $(A)$, which can be thought of 
as the group of isomorphism types of Morita self-equivalences of $A$-Mod. Clearly, any such Morita self-equivalence induces category equivalences

$$
C(A \text {-Mod }) \rightarrow C(A \text {-Mod }), \quad C^{b}(A \text {-mod }) \rightarrow C^{b}(A \text {-mod }),
$$

and

$$
D(A \text {-Mod }) \rightarrow D(A \text {-Mod }), \quad D^{b}(A \text {-mod }) \rightarrow D^{b}(A \text {-mod }) .
$$

This provides us with a natural action of $\operatorname{Pic}(A)$, and consequently also of $\operatorname{Out}(A)$, on sets of isomorphism classes of objects in $C(A$-Mod) (resp. $D(A$-Mod) $)$. Given any complex $\mathbf{X} \in C(A$-Mod $)$, we will accordingly consider its Out-orbit

$$
\left\{{ }^{\sigma} \mathbf{X} \in C(A \text {-Mod }): \bar{\sigma} \in \operatorname{Out}(A)\right\}
$$

in $C(A$-Mod $)$, and analogously that in $D(A$-Mod $)$, as well as its Out-stabilizer

$$
\operatorname{Out}^{\mathbf{X}}(A)=\left\{\bar{\sigma} \in \operatorname{Out}(A):{ }^{\sigma} \mathbf{X} \cong \mathbf{X} \text { in } D(A \text {-mod })\right\}
$$

in the derived category. As we are dealing with bimodule complexes ${ }_{A} \mathbf{X}_{B}$, it will promote orientation to denote the actions of $\operatorname{Out}(A)$ and $\operatorname{Out}(B)$ by means of left and right superscripts, respectively.

Proposition 11. Let ${ }_{A} \mathbf{X}_{B}$ be a bounded, faithfully balanced bimodule complex of finite dimensional modules. If the Out-orbits of ${ }_{A} \mathbf{X}$ and $\mathbf{X}_{B}$ in $C^{b}(A$-mod $)$ and $C^{b}\left(B\right.$-mod) are finite, then the groups $\operatorname{Out}(A)^{0}$ and $\operatorname{Out}(B)^{0}$ are isomorphic. In particular, this is the case when both ${ }_{A} \mathbf{X}$ and $\mathbf{X}_{B}$ are almost projective with $\operatorname{Hom}_{D^{b}(A \text {-mod })}(\mathbf{X}, \mathbf{X}[1])=0$ and $\operatorname{Hom}_{D^{b}(\bmod -B)}(\mathbf{X}, \mathbf{X}[1])=0$. If, in addition, char $K=0$, the first Hochschild cohomology groups of $A$ and $B$ are isomorphic as well.

Proof. The first assertion is simply a translation of Corollary 2.6 in [7] into the present context; the proof given in [7. carries over. The second part is an immediate consequence of Corollary 9 of the previous section. Since the Lie algebra associated to $\operatorname{Out}(A)^{0}$ coincides with the first Hochschild cohomology group of $A$ in characteristic 0 , the last assertion follows as well.

Example. The above propositions cover situations which may be far removed from the situation where $A$ and $B$ are derived equivalent. For instance, if $M$ is any nonzero left $A$-module with the properties that $\operatorname{Hom}_{A}(M, A)=0$ and the Out-orbit of $M$ is finite, then, by taking $X=A \oplus M$ and $B=\operatorname{End}\left({ }_{A} X\right)^{\mathrm{op}} \cong\left[\begin{array}{l}A \\ 0 \\ \operatorname{End}_{A}(M)^{\mathrm{op}}\end{array}\right]$ we obtain a faithfully balanced bimodule ${ }_{A} X_{B}$ satisfying the hypotheses of the preceding propositions. Consequently, the centers of $A$ and $B$ are isomorphic, as are $\operatorname{Out}(A)^{0}$ and $\operatorname{Out}(B)^{0}$; the same is true for the first Hochschild cohomology groups of $A$ and $B$, provided that char $K=0$. But $A$ and $B$ fail to be derived equivalent, since they do not have the same number of simple modules. More specific examples of such modules $M$ are the preprojective modules (in the sense of [2]) which are devoid of projective direct summands. In case $\operatorname{Out}(A)$ is finite, all modules $M$ with vanishing $A$-dual $\operatorname{Hom}_{A}(M, A)$ qualify, of course. Finiteness of $\operatorname{Out}(A)$, in turn, is guaranteed whenever $A$ is a split algebra which is tilting-cotilting equivalent to a hereditary algebra of tree type (cf. [7], Theorem 2.10)

Our principal aim is to obtain the conclusion of Proposition 11 in case $A$ and $B$ are derived equivalent algebras. For that purpose, we stretch the concept of a faithfully balanced complex of bimodules as follows. 
Definition. A complex ${ }_{A} \mathbf{X}_{B}$ of $A$-B-bimodules will be called derived faithfully balanced, provided that the canonical $K$-algebra homomorphisms

$$
\lambda: A \longrightarrow \operatorname{End}_{D(\operatorname{Mod}-B)}\left(\mathbf{X}_{B}\right) \text { and } \rho: B \longrightarrow \operatorname{End}_{D(A-\operatorname{Mod})}\left({ }_{A} \mathbf{X}\right)^{\mathrm{op}}
$$

are isomorphisms.

Remark 12. Suppose that ${ }_{A} \mathbf{X}_{B}$ is a derived faithfully balanced bimodule complex. Clearly, the canonical $K$-algebra homomorphism $\rho: B \rightarrow \operatorname{End}_{D(A \text {-Mod })}\left({ }_{A} \mathbf{X}\right)^{\text {op }}$ equals the composition

$$
\rho: B \rightarrow \operatorname{End}_{C(A-\operatorname{Mod})}\left({ }_{A} \mathbf{X}\right)^{\mathrm{op}} \rightarrow \operatorname{End}_{D(A-\mathrm{Mod})}\left({ }_{A} \mathbf{X}\right)^{\mathrm{op}}
$$

where the second map is the canonical one. If we set $\widehat{B}=\operatorname{End}_{C(A-\operatorname{Mod})}\left({ }_{A} \mathbf{X}\right)^{\text {op }}$, our hypothesis on ${ }_{A} \mathbf{X}_{B}$ thus yields a vector space decomposition $\widehat{B}=B \oplus H$, where $H$ is the two-sided ideal of $\widehat{B}$ consisting of the chain maps $h:{ }_{A} \mathbf{X} \rightarrow{ }_{A} \mathbf{X}$ which become zero in $D(A$-Mod $)$. In the special case where ${ }_{A} \mathbf{X}$ is an almost projective complex of left $A$-modules, these chain maps $h$ are precisely the ones which are homotopic to zero.

Of course, the preceding remark has a twin sibling, with the roles of $A$ and $B$ switched. The same is true for the following useful observation.

Lemma 13. Let ${ }_{A} \mathbf{X}_{B}$ be a bounded almost projective complex of $A$-B-bimodules which is derived faithfully balanced. Then there is an idempotent

$$
e \in \operatorname{End}_{C^{b}(A-\text { mod })}\left({ }_{A} \mathbf{X}\right)^{\text {op }}
$$

with the following properties:

(1) ${ }_{A} \mathbf{X}(1-e)$ is an acyclic complex of left $A$-modules; in particular,

$$
B \cong \operatorname{End}_{D^{b}(A \text {-mod })}\left({ }_{A} \mathbf{X} e\right)^{\mathrm{op}} .
$$

(2) The kernel of the canonical K-algebra homomorphism

$$
\operatorname{End}_{C^{b}(A \text {-mod })}\left({ }_{A} \mathbf{X} e\right) \rightarrow \operatorname{End}_{D^{b}(A \text {-mod })}\left({ }_{A} \mathbf{X} e\right)
$$

is contained in the Jacobson radical of $\operatorname{End}_{C^{b}(A \text {-mod })}\left({ }_{A} \mathbf{X} e\right)$.

(3) $A$ chain map in $\operatorname{End}_{C^{b}(A \text {-mod })}\left({ }_{A} \mathbf{X} e\right)$ is an isomorphism in $C^{b}(A$-mod) if and only if it turns into an isomorphism in $D^{b}(A$-mod).

Proof. As in Remark 12, we denote by $\widehat{B}$ the endomorphism ring

$$
\operatorname{End}_{C^{b}(A-\bmod )}\left({ }_{A} \mathbf{X}\right)^{\mathrm{op}}
$$

and by $H$ the twosided ideal of those chain maps that become trivial in $D^{b}(A$-mod). Then $\widehat{B}$ is in turn a finite dimensional algebra, because the complex $\mathbf{X}$ is bounded by hypothesis. The twosided ideal $(H+J(\widehat{B})) / J(\widehat{B})$ of $\widehat{B} / J(\widehat{B})$ therefore gives rise to an idempotent $f \in H+J(\widehat{B})$ such that $H+J(\widehat{B})=f \widehat{B}+J(\widehat{B})=\widehat{B} f+J(\widehat{B})$. We verify that $f$ is trivial on the homology groups of $\mathbf{X}$. Indeed, if we write $f$ in the form $f=h+j$, with $h \in H$ and $j \in J(\widehat{B})$, then $H^{n}(f)=H^{n}(j)$ for all $n$ by Remark 12. But $j$ being nilpotent, so is the induced map $H^{n}(j)$, and hence $H^{n}(f)=0$ due to idempotency. Thus the chain complex $\mathbf{X} f$ is acyclic, and the choice $e=1-f$ satisfies condition (1). Now the kernel $e H e$ of the canonical $K$ algebra homomorphism $e \widehat{B} e \cong \operatorname{End}_{C^{b}(A-\bmod )}\left({ }_{A} \mathbf{X} e\right)^{\mathrm{op}} \rightarrow \operatorname{End}_{D^{b}(A \text {-mod })}\left({ }_{A} \mathbf{X} e\right)^{\mathrm{op}} \cong$ $B$ is contained in $e J(\widehat{B}) e$; for if it were not, $H$ would fail to be contained in $f \widehat{B}+$ $J(\widehat{B})$, contrary to our choice of $f$. To check the final point of our claim, we consider 
the canonical surjection $\operatorname{End}_{C^{b}(A-\bmod )}\left({ }_{A} \mathbf{X} e\right)^{\mathrm{op}} \cong e \widehat{B} e \rightarrow e \widehat{B} e / e H e \rightarrow e \widehat{B} e / e J(\widehat{B}) e$. If $\beta \in \operatorname{End}_{C^{b}(A \text {-mod })}\left({ }_{A} \mathbf{X} e\right)$ is a quasi-isomorphism, i.e., if $\beta$ becomes a unit in $e \widehat{B} e / e H e$, then, a fortiori, $\beta+e J(\widehat{B}) e$ is a unit in $e \widehat{B} e / e J(\widehat{B}) e$. But $J(e \widehat{B} e)=$ $e J(\widehat{B}) e$ being nilpotent, this implies that $\beta$ is a unit in $e \widehat{B} e=\operatorname{End}_{C^{b}(A-\bmod )}\left({ }_{A} \mathbf{X} e\right)^{\text {op }}$, which proves (3).

Our strategy in showing that the isomorphism type of $\operatorname{Out}(A)^{0}$ is invariant under derived equivalence is to focus on a certain closed subgroup of finite index of Out $(A)$; invariance of the identity component of this subgroup will then of course amount to invariance of the identity component of the full outer automorphism group. More precisely, given mutually inverse twosided tilting complexes ${ }_{A} \mathbf{X}_{B}$ and ${ }_{B} \widetilde{\mathbf{X}}_{A}$ in the sense of [15], the subgroups of choice in $\operatorname{Out}(A)$ and $\operatorname{Out}(B)$ will be the stabilizer subgroups $\operatorname{Out}^{\mathbf{X}}(A)$ and $\operatorname{Out}^{\tilde{\mathbf{x}}}(B)$ of $\mathbf{X}$ and $\widetilde{\mathbf{X}}$ in $D^{b}(A$-mod) and $D^{b}(B$-mod), respectively. The construction of an isomorphism between them hinges on the following concept of a 'semilinear chain map' between complexes of left $A$-modules: Fix $\sigma \in \operatorname{Aut}(A)$ and complexes $\mathbf{Y}, \mathbf{Z}$ of left $A$-modules. Following the pattern established in [7], we call a chain map $\varphi=\left(\varphi_{n}\right)_{n \in \mathbb{N}}: \mathbf{Y} \rightarrow \mathbf{Z}$ of $K$-complexes $\sigma$-semilinear, provided that $\varphi_{n}(a y)=a^{\sigma} \varphi_{n}(y)$ for all $n \in \mathbb{N}, y \in Y_{n}$, and $a \in A$. We note that a $\sigma$-semilinear map $\mathbf{Y} \rightarrow \mathbf{Z}$ can alternately be viewed as a morphism ${ }^{\sigma^{-1}} \mathbf{Y} \rightarrow \mathbf{Z}$ in $C(A$-Mod). Moreover, we observe that, given any $\sigma$-semilinear automorphism $\varphi$ of $\mathbf{Y}$ and any morphism $\beta \in \operatorname{End}_{C(A \text {-Mod })}(\mathbf{Y})^{\mathrm{op}}$, the conjugate $\varphi \beta \varphi^{-1}$ is again a morphism in $\operatorname{End}_{C(A-M o d)}(\mathbf{Y})^{\mathrm{op}}$. The endomorphisms (resp., automorphisms) of the $K$-complex $\mathbf{Y}$ which are $\sigma$-semilinear for some $\sigma \in \operatorname{Aut}(A)$ are collectively referred to as the $(A$-) semilinear endomorphisms (resp., automorphisms) of $\mathbf{Y}$. It is straightforward that the set $\mathcal{S}(\mathbf{Y})$ of all semilinear automorphisms of $\mathbf{Y}$ forms a subgroup of the group of automorphisms of the underlying $K$-complex.

In the following $\stackrel{L}{\otimes}$ will denote the left derived functor of the tensor functor on chain complexes. Keep in mind that, under the hypothesis of the following lemma, the algebra $e \widehat{B} e / e H e$ is canonically isomorphic to $B$.

Lemma 14. Again, let ${ }_{A} \mathbf{X}_{B}$ be a bounded almost projective complex of $A-B$ bimodules, which is derived faithfully balanced, let $e \in \operatorname{End}_{C^{b}(A \text {-mod })}\left({ }_{A} \mathbf{X}\right)^{\mathrm{op}}$ be chosen as in Lemma 13, and let $H$ be the twosided ideal of

$$
\widehat{B}=\operatorname{End}_{C^{b}(A \text {-mod })}\left({ }_{A} \mathbf{X}\right)^{\mathrm{op}}
$$

as specified in Remark 12. Given automorphisms $\sigma \in \operatorname{Aut}(A)$ and $\tau \in \operatorname{Aut}(B)$, conditions (1)-(3) below are related as follows: '(1) $\Longrightarrow(2)^{\prime}$ ' and '(2) $\Longleftrightarrow(3)$ '.

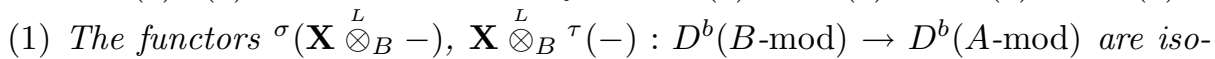
morphic.

(2) There is a $\sigma$-semilinear chain map

$$
\xi: \mathbf{X} \rightarrow \mathbf{X}
$$

i.e., a map in $\operatorname{Hom}_{C^{b}(A \text {-mod })}\left(\sigma^{-1} \mathbf{X}, \mathbf{X}\right)$, which turns into an isomorphism ${ }^{\sigma^{-1}} \mathbf{X} \rightarrow$ $\mathbf{X}$ in $D^{b}\left(A\right.$-mod) and which satisfies the condition that $\xi b=b^{\tau} \xi$ in $D^{b}(A$-mod), for all $b \in B$.

(3) There is a bijective $\sigma$-semilinear chain map $\varphi: \mathbf{X} e \rightarrow \mathbf{X} e$ such that the automorphism of $e \widehat{B}$, given by $\beta \mapsto \varphi \beta \varphi^{-1}$, induces the same map as $\tau$ on $B$. 
Proof. (1) $\Longrightarrow(2)$. One readily verifies that (1) is equivalent to the functors $\sigma^{-1}\left(\mathbf{X} \stackrel{L}{\otimes}_{B}-\right)$ and $\mathbf{X} \stackrel{L}{\otimes}_{B} \tau^{-1}(-)$ being isomorphic. We assume that this latter condition is satisfied, and recall that, when applied to stalk complexes of projectives, the functor $\mathbf{X}^{L} \otimes_{B}-$ is just the usual tensor product (cf. [18], ch. 2). Now the tensor products $\mathbf{X} \otimes_{B}\left({ }^{\tau^{-1}} B\right)$ and $\mathbf{X}^{\tau} \otimes_{B} B$ are clearly canonically isomorphic; we will identify them in fact. Specifying an isomorphism $\chi:{ }^{-1}\left(\mathbf{X}^{L} \otimes_{B}-\right) \rightarrow \mathbf{X}^{L} \otimes_{B}{ }^{\tau^{-1}}(-)$ of functors $D^{b}(B$-mod $) \rightarrow D^{b}(A$-mod $)$, we consider $\chi(B):{ }^{\sigma^{-1}}\left(\mathbf{X} \otimes_{B} B\right) \rightarrow \mathbf{X} \otimes_{B}$ $\tau^{-1} B$ and the resulting chain of isomorphisms in $D^{b}(A$-mod):

$$
{ }^{\sigma^{-1}} \mathbf{X} \cong \sigma^{-1}\left(\mathbf{X} \otimes_{B} B\right) \cong \mathbf{X} \otimes_{B}{ }^{\tau^{-1}} B=\mathbf{X}^{\tau} \otimes_{B} B \cong \mathbf{X}^{\tau} .
$$

Since ${ }^{\sigma^{-1}} \mathbf{X}$ and $\mathbf{X}^{\tau}$ are both almost projective complexes of left $A$-modules, the displayed isomorphism is, on the level of the derived categories, induced by a chain $\operatorname{map} \xi:{ }^{-1} \mathbf{X} \rightarrow \mathbf{X}^{\tau}$ in $C^{b}(A$-mod) (see the remarks following the definition of an almost projective complex in Section 1). Alternately expressed, $\xi$ is a $\sigma$-semilinear map $\mathbf{X} \rightarrow \mathbf{X}^{\tau}$ which becomes bijective in $D^{b}(A$-mod). Finally, the automorphism $\tau$ of $A$ makes a relevant appearance: Namely, the naturality of $\chi$ translates into the required additional property of $\xi$.

$(2) \Longrightarrow(3)$. Let $\xi: \mathbf{X} \rightarrow \mathbf{X}$ be a $\sigma$-semilinear map as specified in (2). The fact that the morphism $\mathbf{X} \stackrel{e}{\rightarrow} \mathbf{X} e$ turns into an isomorphism in $D^{b}(A$-mod) implies that, in the derived category, $\xi$ can be viewed as an isomorphism ${ }^{\sigma^{-1}} \mathbf{X} e \rightarrow \mathbf{X} e$, induced by the chain map $\varphi=e \xi\left(\left.e\right|_{\mathbf{X} e}\right):{ }^{-1} \mathbf{X} e \rightarrow \mathbf{X} e$ in $C^{b}(A$-mod). From Lemma 13, part (3), one easily derives that $\varphi$ is actually an isomorphism in $C^{b}(A$-mod). Thus $\varphi$ is a $\sigma$-semilinear automorphism $\mathbf{X} e \rightarrow \mathbf{X} e$. Checking the remaining condition under (3) is routine.

$(3) \Longrightarrow(2)$. Using the equality $\mathbf{X}=\mathbf{X} e \oplus \mathbf{X}(1-e)$, one extends $\varphi: \mathbf{X} e \rightarrow \mathbf{X} e$ to a $\sigma$-semilinear map $\xi=\varphi \oplus 0: \mathbf{X} \rightarrow \mathbf{X}$ and notes that, trivially, the extension has the required properties.

The following proposition represents the second crucial step - next to Corollary 9 - on the road towards the main result of this section. We continue to study a complex $\mathbf{X}$ of $A$ - $B$-bimodules as in Lemma 14, still denoting its endomorphism ring in $C^{b}(A$-mod) by $\widehat{B}$. Moreover, we introduce a set $\mathcal{G}$ which will turn out to be the graph of a group isomorphism $\phi: \operatorname{Out}^{\mathbf{X}}(A) \rightarrow \operatorname{Out}^{\tilde{\mathbf{x}}}(B)$, provided that ${ }_{A} \mathbf{X}_{B}$ and ${ }_{B} \widetilde{\mathbf{X}}_{A}$ are mutually inverse twosided tilting complexes. Namely, we define $\mathcal{G}$ as the set of all pairs $(\bar{\sigma}, \bar{\tau}) \in \operatorname{Out}(A) \times \operatorname{Out}(B)$ such that $\sigma$ and $\tau$ satisfy the equivalent conditions (2), (3) of Lemma 14. Since, from the resulting definition of $\phi$, it is not obvious that this isomorphism of abstract groups is actually an isomorphism of algebraic groups, we resort to viewing $\phi$ from an alternate angle. Namely, we will rewrite $\phi$ in the form $p_{2} p_{1}^{-1}$, with isomorphisms $p_{1}: \mathcal{S} \rightarrow \operatorname{Out}^{\mathbf{x}}(A)$ and $p_{2}: \mathcal{S} \rightarrow \operatorname{Out}^{\tilde{\mathbf{x}}}(B)$ for a suitable choice of $\mathcal{S}$, these latter maps having the benefit of being more readily recognizable as isomorphisms of algebraic groups. To that end, we consider the group $\mathcal{S}(\mathbf{X} e)$ of semilinear automorphisms of the complex $\mathbf{X} e \in C^{b}(A$-mod), together with the following two subgroups $\mathcal{U}$ and $\mathcal{V}$ : The subgroup $\mathcal{U}$ consists of the maps of the form $x \mapsto u x v$, where $u$ is a unit of $A$ and $v$ a unit of $e \widehat{B} e$ (note that the latter assignment is semilinear with respect to the automorphism $a \mapsto u a u^{-1}$ of $A$ ). The subgroup $\mathcal{V}$ of $\mathcal{S}(\mathbf{X} e)$ consists of those maps $\varphi \in \mathcal{S}(\mathbf{X} e)$ for which the algebra automorphism $e \widehat{B} e \rightarrow e \widehat{B} e$, given by $\beta \rightarrow \varphi \beta \varphi^{-1}$, 
induces an inner automorphism on $e \widehat{B} e / e H e \cong B$; here again, $H \subseteq \widehat{B}$ is the ideal of those chain maps which vanish in the derived category $D^{b}(A$-mod). One readily checks that $\mathcal{U}$ and $\mathcal{V}$ are normal subgroups of $\mathcal{S}(\mathbf{X} e)$ and that $\mathcal{U}$ is contained in $\mathcal{V}$. This provides us with a canonical projection $\pi: \mathcal{S}(\mathbf{X} e) / \mathcal{U} \rightarrow \mathcal{S}(\mathbf{X} e) / \mathcal{V}$.

Proposition 15. For any bounded almost projective complex ${ }_{A} \mathbf{X}_{B}$ of $A$-B-bimodules which is derived faithfully balanced, the following are true:

(1) The set $\mathcal{G}$ is the graph of a group homomorphism $\phi: \operatorname{Out}^{\mathbf{X}}(A) \rightarrow \operatorname{Out}(B)$, where $\operatorname{Out}^{\mathbf{X}}(A)$ is the $\operatorname{Out}(A)$-stabilizer of $\mathbf{X}$ in $D^{b}(A$-mod).

(2) In case $\mathbf{X}$ is a tilting complex over $A$ and $e \in \operatorname{End}_{C^{b}(A-\bmod )}(\mathbf{X})^{\text {op }}$ an idempotent as before, the subgroups $\mathcal{U}$ and $\mathcal{V}$ of $\mathcal{S}(\mathbf{X} e)$ are closed, as is the subgroup $\operatorname{Im}(\phi)$ of $\operatorname{Out}(B)$. Moreover, there are isomorphisms of algebraic groups,

$$
p_{1}: \mathcal{S}(\mathbf{X} e) / \mathcal{U} \rightarrow \operatorname{Out}^{\mathbf{X}}(A) \quad \text { and } \quad p_{2}: \mathcal{S}(\mathbf{X} e) / \mathcal{V} \rightarrow \operatorname{Im}(\phi),
$$

such that $p_{2} \pi=\phi p_{1}$, where $\pi$ is the projection introduced above.

Proof. (1) The task of checking that an element $\bar{\sigma} \in \operatorname{Out}(A)$ cannot be the first component of two different pairs in $\mathcal{G}$ is easily reduced to the case where $\bar{\sigma}=1$, i.e., to the case where $\sigma(a)=u a u^{-1}$ for some unit $u$ of $A$. Suppose that $\tau \in$ $\operatorname{Aut}(B)$ is such that the pair $(\sigma, \tau)$ satisfies the equivalent conditions of Lemma 14, and let $\varphi: \mathbf{X} e \rightarrow \mathbf{X} e$ be a bijective $\sigma$-semilinear endomorphism of $\mathbf{X} e$ as specified in condition (3) of that lemma; this is to say that $\beta \mapsto \varphi \beta \varphi^{-1}$ induces the same map as $\tau$ on $e \widehat{B} e / e H e$. The $\sigma$-semilinearity of $\varphi$ yields $A$-linearity of the assignment $v: \mathbf{X} e \rightarrow \mathbf{X} e$ defined by $x v=u^{-1}(x \varphi)$. In other words, $v$ is a unit in $\operatorname{End}_{C^{b}(A \text {-mod })}(\mathbf{X} e)^{\mathrm{op}}=e \widehat{B} e$. Keeping in mind that the algebra $e \widehat{B} e / e H e$ is isomorphic to $B$ by our balancedness hypothesis and the choice of $H$, we let $c$ be the canonical image of $v$ in $B$. In view of the equality $v \beta v^{-1}=\varphi \beta \varphi^{-1}$ for $\beta \in e \widehat{B} e$, we finally observe that $\tau$ is just conjugation by $c$, for our construction entails $\tau(b)=\varphi b \varphi^{-1}=c b c^{-1}$ for $b \in B$. Thus $\tau$ is in turn inner, as required.

Knowing that $\mathcal{G}$ is the graph of a function $\phi$, we pin down the domain of $\phi$. By construction, it consists of those classes $\bar{\sigma} \in \operatorname{Out}(A)$ for which there exists a bijective $\sigma$-semilinear map $\varphi: \mathbf{X} e \rightarrow \mathbf{X} e$ (note that conjugation by $\varphi$ automatically induces an automorphism of $e \widehat{B} e / e H e)$. The latter condition is tantamount to the requirement that $\mathbf{X} e$ be isomorphic to ${ }^{\sigma}(\mathbf{X} e)$ in $C^{b}(A$-mod), which is in turn equivalent to the existence of an isomorphism $\mathbf{X} e \cong \sigma(\mathbf{X} e)$ in $D^{b}(A$-mod); this last equivalence is readily deduced from Lemma $13(3)$. So the domain of $\phi$ is $\operatorname{Out}^{\mathbf{X}}(A)$, as claimed. It is straightforward to check that $\phi$ is a group homomorphism.

(2) Now suppose that $\mathbf{X}$ is a tilting complex of left A-modules. In particular, this means that the category add $(\mathbf{X})$, consisting of the finite direct sums of direct summands of copies of $\mathbf{X}$, generates the homotopy category $K^{b}(A$-proj) of all bounded projective complexes of finitely generated left $A$-modules as a triangulated category. We infer that the left annihilator of $\mathbf{X}$ in $A$ is zero. Hence so is the annihilator of $\mathbf{X} e$, for the projective complexes $\mathbf{X}$ and $\mathbf{X} e$ coincide in $K^{b}(A$-proj). Following the model of [7] Lemma 1.2], we deduce that, for every semilinear bijective chain map $\varphi: \mathbf{X} e \rightarrow \mathbf{X} e$, there is a unique automorphism $\sigma \in \operatorname{Aut}(A)$ with the property that $\varphi$ is $\sigma$-semilinear: Namely, if $\ell_{a}$ denotes left multiplication of $\mathbf{X} e$ by $a$, then $\sigma(a)$ is determined by the requirement that $\ell_{\sigma(a)}=\varphi \ell_{a} \varphi^{-1}$. If we write $\mathcal{S}$ for the group of semilinear automorphisms of $\mathbf{X} e$, this provides us with a map $\mathcal{S} \rightarrow \operatorname{Aut}(A)$, which is actually a homomorphism of abstract groups. The proof of assertion (1) shows that, whenever this homomorphism takes $\varphi$ to an inner automorphism ' $a \mapsto u a u^{-1}$, 
for a suitable unit $u \in A$, the map $v: \mathbf{X} e \rightarrow \mathbf{X} e$ sending $x$ to $u^{-1}(x \varphi)$ is a unit in $e \widehat{B} e$, and vice versa. But the latter condition just says that $x \varphi=u x v$, and thus shows that our group homomorphism $\mathcal{S} \rightarrow \operatorname{Aut}(A)$ induces a monomorphism $\mathcal{S} / \mathcal{U} \rightarrow \operatorname{Out}(A)$ of abstract groups. In light of the previous paragraph, the image of this monomorphism is $\operatorname{Out}^{\mathbf{x}}(A)$, and hence it gives rise to an isomorphism $p_{1}: \mathcal{S} / \mathcal{U} \rightarrow \operatorname{Out}^{\mathbf{x}}(A)$.

Set $p_{2}^{\prime}=\phi p_{1}: \mathcal{S} / \mathcal{U} \rightarrow \operatorname{Out}(B)$. Then, clearly, $\operatorname{Im}\left(p_{2}^{\prime}\right)=\operatorname{Im}(\phi)$. As for the kernel of $p_{2}^{\prime}$, we have $\phi p_{1}(\varphi+\mathcal{U})=1$ if and only if there is an inner automorphism $\iota$ of $B$ such that $b^{\iota} \varphi$ equals $\varphi b$ in $D^{b}(A$-mod), for all $b \in B$. But this just means that the automorphism of $e \widehat{B} e$ given by $\beta \rightarrow \varphi \beta \varphi^{-1}$ induces an inner automorphism of the algebra $e \widehat{B} e / e H e \cong B$. Hence $\operatorname{Ker}\left(p_{2}^{\prime}\right)$ equals $\mathcal{V} / \mathcal{U}$, so that $p_{2}^{\prime}$ induces an isomorphism $\mathcal{S} / \mathcal{V} \rightarrow \operatorname{Im}(\phi)$, as required.

Finally, we need to ascertain that $p_{1}, p_{2}$ are isomorphisms of algebraic groups. First, for $p_{1}$, we start by identifying the algebra $A$ with the subalgebra of $\widehat{A}=$ End $_{C^{b}(\text { mod- } e \hat{B} e)}(\mathbf{X} e)$ consisting of the left multiplications $\ell_{a}$ on $\mathbf{X} e$ for $a \in A$; this identification is legitimate, since $\mathbf{X} e$ is faithful over $A$. With $A$ viewed from this angle, the group homomorphism $\mathcal{S} \rightarrow \operatorname{Aut}(A)$ underlying $p_{1}$ maps $\varphi \in \mathcal{S}$ to the automorphism $\ell_{a} \mapsto \varphi \ell_{a} \varphi^{-1}$ of $A$, the latter conjugate being equal to $\ell_{\sigma(a)}$ if $\varphi$ is $\sigma$-semilinear. This assignment is clearly a morphism of algebraic groups, and hence $\mathcal{U}$ is a closed (normal) subgroup of $\mathcal{S}$. To see that the inverse of $p_{1}$ is again a morphism, it suffices to show that the map $p_{1}^{\prime}: \mathcal{S} \rightarrow$ Out $^{\mathbf{x}}(A)$ which induces $p_{1}$ modulo $\mathcal{U}$ has a surjective differential $T_{e}(\mathcal{S}) \rightarrow T_{e}\left(\operatorname{Im}\left(p_{1}^{\prime}\right)\right.$ ) (cf. [3, Proposition $6.13])$.

In a first step, we focus on the following morphism of algebraic groups:

$$
\gamma: G \rightarrow \mathrm{GL}(V) \quad \text { with } \quad \gamma_{g}(v)=g v g^{-1},
$$

where $G=\mathrm{GL}(\mathbf{X} e)$ and $V=\operatorname{End}_{K}(\mathbf{X} e)$. Our aim is to see that the restriction $\mathcal{S} \rightarrow \gamma(\mathcal{S})$, again denoted by $\gamma$, has surjective differential d $\gamma: T_{e}(\mathcal{S}) \rightarrow T_{e}(\operatorname{Im}(\gamma)) \subseteq$ $\operatorname{End}_{K}(V)$. From [3, 3.10], we know that

$$
(\mathrm{d} \gamma)(X)(v)=\left(\mathrm{d}_{\mathfrak{o r b}}\right)_{e}(X)
$$

for $X \in T_{e}(\mathcal{S})$ and $v \in V$, where $\mathfrak{o r b}_{v}: \mathcal{S} \rightarrow \gamma(\mathcal{S}) v$ is the orbit map $g \mapsto \gamma_{g} v$. To check surjectivity, we let $v_{1}, \ldots, v_{n}$ be a basis for $V$, set $\underline{v}=\left(v_{1}, \ldots, v_{n}\right) \in V^{n}$, and consider the 'expanded orbit map'

$$
\underline{\mathfrak{O} \mathfrak{r b}}: G \rightarrow \gamma(G) \cdot \underline{v}, \quad g \mapsto g \cdot \underline{v}=\left(\gamma_{g} v_{1}, \ldots, \gamma_{g} v_{n}\right),
$$

and its restriction to $\mathcal{S}$,

$$
\underline{\mathfrak{o r b}}: \mathcal{S} \rightarrow \gamma(\mathcal{S}) . \underline{v} .
$$

The map $\underline{\mathfrak{O} \mathfrak{r} \mathfrak{b}}$ has reduced fibres, since the stabilizer subgroup $\operatorname{Stab}(\underline{v})$ of $\underline{v}$ in $G$ clearly arises as the solution set of a system of linear equations (for background on reduced fibres, see [10 A.I.2.5, 2.6]). By [10, A.I.5.5, Satz 2], this implies that $\operatorname{Ker}(\mathrm{d} \underline{\mathfrak{O} \mathfrak{r} \mathfrak{b}})_{e}=T_{\underline{v}}(\operatorname{Stab}(\underline{v}))$. Cutting down $\operatorname{Stab}(\underline{v})$ to the stabilizer subgroup $\operatorname{stab}(\underline{v})$ of $\underline{v}$ in $\mathcal{S}$, we infer that $\operatorname{Ker}(\mathrm{d} \underline{\mathfrak{o r b} b})_{e}$ equals $T_{\underline{v}}(\operatorname{stab}(\underline{v}))$, and, in view of [3, Proposition 6.7], we deduce further that the differential $(\mathrm{d} \underline{\mathfrak{o r b}})_{e}: T_{e}(\mathcal{S}) \rightarrow$ $T_{\underline{v}}(\gamma(\mathcal{S}) . \underline{v})$ is surjective. In light of $(\dagger)$, this yields surjectivity of $\overline{\mathrm{d} \gamma}$. 
If we enlarge the codomain of $p_{1}^{\prime}$ from $\operatorname{Out}^{\mathbf{x}}(A)$ to $\operatorname{Out}(A)$, then $p_{1}^{\prime}$ can be factored in the form

$$
\mathcal{S} \stackrel{\gamma}{\longrightarrow} \gamma(\mathcal{S}) \stackrel{\mathfrak{r e s}}{\longrightarrow} \operatorname{Aut}(A) \stackrel{\mathfrak{c a n}}{\longrightarrow} \operatorname{Out}(A),
$$

where $\mathfrak{r e s}: \gamma(\mathcal{S}) \rightarrow \operatorname{Aut}(A)$ sends any $K$-automorphism of $V$ in $\gamma(\mathcal{S})$ to its restriction to the invariant subspace $A=\widehat{A}$. Now $\mathfrak{r e s}: \gamma(\mathcal{S}) \rightarrow \operatorname{Im}(\mathfrak{r e s})$ is clearly separable, and so is the canonical map $\mathfrak{c a n}$, the latter being actually a geometric quotient; hence both $\mathfrak{r e s}$ and $\mathfrak{c a n}$ have surjective differentials. This shows that our auxiliary map $p_{1}^{\prime}$ has surjective differential, and we conclude that $p_{1}$ is indeed an isomorphism of algebraic groups.

To deal with $p_{2}$, we identify $B$ with $e \widehat{B} e / e H e$ via the canonical isomorphism. Clearly, the group homomorphism $\mathcal{S} \rightarrow \operatorname{Aut}(e \widehat{B} e)$ sending $\varphi$ to the automorphism $\beta \mapsto \varphi \beta \varphi^{-1}$ respects the variety structures, and since the normal subgroup of $\operatorname{Aut}(e \widehat{B} e)$ consisting of those automorphisms which induce inner automorphisms of $e \widehat{B} e / e H e$ is closed, this subgroup gives rise to a geometric quotient of $\operatorname{Aut}(e \widehat{B} e)$. In particular, the auxiliary map $p_{2}^{\prime}: \mathcal{S} / \mathcal{U} \rightarrow \operatorname{Out}(B)$ as above is again a morphism of algebraic groups. This in turn entails that $\mathcal{V} \subseteq \mathcal{S}$ and $\operatorname{Im}(\phi) \subseteq \operatorname{Out}(B)$ are closed subgroups. Now the geometric quotient property of the canonical map $\mathcal{S} \rightarrow \mathcal{S} / \mathcal{V}$ guarantees that $p_{2}$ is a morphism of algebraic groups as well. With an argument similar to that given for $p_{1}$, one finally shows that $p_{2}$ also has a surjective differential, which guarantees that its inverse is in turn a morphism of algebraic groups.

In the following, we adopt Rickard's terminology in [15], but insignificantly deviate from his conventions by calling a category equivalence $D^{-}(B$-Mod $) \rightarrow$ $D^{-}\left(A\right.$-Mod) (resp., $D^{b}(B$-mod $) \rightarrow D^{b}(A$-mod $\left.)\right)$ standard if it is isomorphic to $\mathbf{X} \stackrel{L}{\otimes}_{B}-$, where ${ }_{A} \mathbf{X}_{B}$ is a two-sided tilting complex; as is shown in [15. Theorem 3.3], this is harmless. Moreover, we note that according to [15, loc. cit.], any equivalence $\mathbf{X} \stackrel{L}{\otimes}_{B}$ - between the derived categories $D^{-}(B$-Mod $)$ and $D^{-}(A$-Mod $)$ automatically restricts to an equivalence $D^{b}(B$-mod $) \rightarrow D^{b}(A$-mod $)$. To finally clear the road to the main theorem of this section, we record an easy subsidiary observation.

Lemma 16. Let $B$ be a finite dimensional algebra and

$$
F: D^{-}(B-\mathrm{Mod}) \rightarrow D^{-}(B-\mathrm{Mod})
$$

a standard derived equivalence. Then the following assertions are equivalent:

(1) $F$ is induced by an element $\bar{\tau} \in \operatorname{Out}(B)$, i.e., there is a natural isomorphism $F \cong \tau(-)$.

(2) $F$ takes the stalk complex ${ }_{B} B$ to an isomorphic copy of itself.

Proof. Clearly, any $\tau \in \operatorname{Aut}(B)$ gives rise to an isomorphism $B \cong{ }^{\tau} B$ of left $B$ modules, which proves '(1) $\Longrightarrow(2)$ '. For the converse, let ${ }_{B} \mathbf{T}_{B}$ be a two-sided tilting complex for $B$ such that $F \cong \mathbf{T} \stackrel{L}{\otimes}_{B}-$. Then $B \cong F(B) \cong \mathbf{T} \stackrel{L}{\otimes} B \cong \mathbf{T}$ in $D^{-}(B$-Mod). In other words, as a complex of left $B$-modules, $\mathbf{T}$ is isomorphic to the stalk complex $B$ on the level of the derived category. By [15], the right derived functor of $\operatorname{Hom}_{B}(\mathbf{T},-)$ is a quasi-inverse of $F-$ call it $F^{-1}$. We deduce that $F^{-1}=R \operatorname{Hom}_{B}(\mathbf{T},-) \cong \operatorname{Hom}_{D^{-}(B-\operatorname{Mod})}(B,-)$ is induced by a Morita equivalence $\operatorname{Hom}_{B}(C,-): B$-Mod $\rightarrow B$-Mod, where $C$ is some $B$ - $B$-bimodule isomorphic to ${ }^{1} B^{\tau^{-1}}$ for some automorphism $\tau$ of $B$. This shows that $F \cong \tau(-)$ is determined by $\bar{\tau} \in \operatorname{Out}(B)$, and completes the proof of '(2) $\Longrightarrow(1)$ '. 
As announced, we will exploit the scenario we have rigged up in the special case where ${ }_{A} \mathbf{X}_{B}$ is a twosided tilting complex. In particular, we will see that, in this situation, the map $\phi$ constructed in Proposition 15 is a group isomorphism Out $^{\mathbf{X}}(A) \rightarrow$ Out $^{\tilde{\mathbf{X}}}(B)$, provided that ${ }_{B} \widetilde{\mathbf{X}}_{A}$ is a twosided tilting complex inverse to $\mathbf{X}$. Moreover, we will obtain $\mathcal{U}=\mathcal{V}$, which will provide us with an alternate description of $\phi$ as $p_{2} p_{1}^{-1}$, in the terminology of the proposition.

Theorem 17. Suppose that $A$ and $B$ are finite dimensional algebras (over an algebraically closed field $K)$. If $A$ and $B$ are derived equivalent, then $\operatorname{Out}(A)^{0}$ and $\operatorname{Out}(B)^{0}$ are isomorphic algebraic groups.

Proof. We begin by choosing two-sided tilting complexes ${ }_{A} \mathbf{X}_{B}$ and ${ }_{B} \widetilde{\mathbf{X}}_{A}$ which are inverse to each other, that is, $\mathbf{X} \stackrel{L}{\otimes}_{B} \widetilde{\mathbf{X}} \cong{ }_{A} A_{A}$ and $\widetilde{\mathbf{X}} \stackrel{\otimes}{\otimes}_{A} \mathbf{X} \cong{ }_{B} B_{B}$ in the derived categories $D^{b}\left(A \otimes_{K} A^{\text {op }}\right.$-mod $)$ and $D^{b}\left(B \otimes_{K} B^{\text {op }}\right.$-mod $)$, respectively. Recall that Out $^{\mathbf{X}}(A)$ and Out ${ }^{\tilde{\mathbf{x}}}(B)$ denote the subgroups consisting of those elements of Out $(A)$ and $\operatorname{Out}(B)$ which stabilize $\mathbf{X}$ and $\widetilde{\mathbf{X}}$ in $D^{b}(A$-mod $)$ and $D^{b}(B$-mod), respectively. Our first goal is to show that these closed subgroups of $\operatorname{Out}(A)$ and $\operatorname{Out}(B)$ have finite index in the corresponding full outer automorphism groups.

We show finiteness of the index $\left[\operatorname{Out}(A): \mathrm{Out}^{\mathbf{X}}(A)\right]$, the result for $B$ being symmetric. Note that the $\operatorname{Out}(A)$-stabilizer of $\mathbf{X}$ in $D^{b}(A$-mod) equals that of $\mathbf{X} e$ in $D^{b}(A$-mod $)$, where $e$ is an idempotent in the $C^{b}(A$-mod $)$-endomorphism ring of $\mathbf{X}$ as specified in Lemma 13, for, in the derived category, $\mathbf{X}$ and $\mathbf{X} e$ become isomorphic. Hence the index under consideration coincides with $\left[\operatorname{Out}(A): \operatorname{Out}^{\mathbf{X} e}(A)\right]$ and thus equals the cardinality of the $\operatorname{Out}(A)$-orbit of $\mathbf{X} e$ in $D^{b}(A$-mod). So clearly it suffices to show that the $\operatorname{Out}(A)$-orbit of $\mathbf{X} e$ in $C^{b}(A-\bmod )$ is finite. But this follows from Corollary 9 of Section 1 , which shows that there are only finitely many complexes over $A$ which share the $K$-dimension of $\mathbf{X} e$, as well as the property that $\operatorname{Hom}_{D^{b}(A-\bmod )}(\mathbf{X} e, \mathbf{X} e[1])=0$. This takes us to our first goal.

Our argument is thus reduced to showing that $\operatorname{Im}(\phi)=\operatorname{Out}^{\tilde{\mathbf{x}}}(B)$ and $\mathcal{U}=$ $\mathcal{V}$, in the terminology of Proposition 15. Once these equalities are established, Proposition 15 will provide us with isomorphisms of algebraic groups

$$
\operatorname{Out}^{\mathbf{X}}(A) \stackrel{p_{1}}{\longleftarrow} \mathcal{S}(\mathbf{X} e) / \mathcal{U} \stackrel{p_{2}}{\longrightarrow} \operatorname{Out}^{\tilde{\mathbf{x}}}(B) .
$$

We deduce that $\phi=p_{2} p_{1}^{-1}$ restricts to an isomorphism $\operatorname{Out}(A)^{0} \rightarrow \operatorname{Out}(B)^{0}$, for the identity component of $\mathcal{S}(\mathbf{X} e) / \mathcal{U}$ is mapped onto that of Out ${ }^{\mathbf{x}}(A)$ - which equals $\operatorname{Out}(A)^{0}-$ by $p_{1}$, and onto that of $\operatorname{Out}^{\tilde{\mathbf{x}}}(B)-$ which equals $\operatorname{Out}(B)^{0}-$ by $p_{2}$.

We access the two remaining equalities by way of the group homomorphism $\phi$ from $\operatorname{Out}^{\mathbf{X}}(A)$ to $\operatorname{Out}(B)$ constructed in Proposition 15. To that end, we give yet another description of $\phi$ in the present situation. For any element $\bar{\sigma} \in \operatorname{Out}^{\mathbf{X}}(A)$, the equivalence $F=\left(\widetilde{\mathbf{X}} \otimes_{A}^{L}-\right) \circ^{\sigma}\left(\mathbf{X}^{\otimes_{B}}{ }_{B}-\right)$ from $D^{b}(B$-mod $)$ to $D^{b}(B$-mod $)$ preserves the isomorphism type of the stalk complex ${ }_{B} B$, whence Lemma 16 shows $F$ to be induced by some element $\bar{\tau} \in \operatorname{Out}(B)$. In view of the choice of $\mathbf{X}$ and $\widetilde{\mathbf{X}}$ as inverse to each other, this provides us with an isomorphism $\sigma\left(\mathbf{X} \stackrel{L}{\otimes}_{B}-\right) \cong \mathbf{X} \stackrel{L}{\otimes}_{B}^{\tau}(-)$ of functors $D^{b}(B$-mod $) \rightarrow D^{b}(A$-mod $)$. Consequently, Lemma 14 guarantees that the pair $(\bar{\sigma}, \bar{\tau})$ satisfies the equivalent conditions (2), (3) of that lemma; in other words, the pair $(\bar{\sigma}, \bar{\tau})$ belongs to the graph $\mathcal{G}$ of $\phi$. Thus $\phi$ assigns to any $\bar{\sigma} \in$ Out $^{\mathbf{X}}(A)$ the unique element $\bar{\tau} \in \operatorname{Out}(B)$ with the property that ${ }^{\sigma}\left(\mathbf{X}^{\otimes^{L}}{ }_{B}-\right) \cong \mathbf{X} \stackrel{L}{\otimes}_{B}{ }^{\tau}(-)$. 
To obtain $\operatorname{Im}(\phi) \subseteq$ Out $^{\tilde{\mathbf{x}}}(B)$, we derive the following string of isomorphisms in $D^{b}(B$-mod):

$$
\begin{aligned}
{ }^{\tau} \widetilde{\mathbf{X}} & \cong\left(\widetilde{\mathbf{X}} \otimes^{L}{ }_{A} \mathbf{X}\right) \stackrel{\perp}{\otimes}_{B}{ }^{\tau} \widetilde{\mathbf{X}} \cong \widetilde{\mathbf{X}} \otimes^{L}\left(\mathbf{X} \otimes_{B}^{L}{ }^{\tau} \widetilde{\mathbf{X}}\right) \\
& \cong \widetilde{\mathbf{X}} \stackrel{\otimes}{ }_{A} \sigma\left(\mathbf{X} \stackrel{\otimes}{ }_{B} \widetilde{\mathbf{X}}\right) \cong \widetilde{\mathbf{X}} \stackrel{\otimes}{\otimes}_{A}{ }^{\sigma} A .
\end{aligned}
$$

Since the left $A$-modules ${ }^{\sigma} A$ and $A$ are isomorphic, we conclude that ${ }^{\tau} \widetilde{\mathbf{X}} \cong \widetilde{\mathbf{X}}$ in $D^{b}\left(B\right.$-mod). But this just means that $\bar{\tau} \in \mathrm{Out}^{\tilde{\mathbf{x}}_{(}}(B)$.

The symmetric version of Proposition 15, obtained by switching the roles of $A$ and $B$, permits us to 'symmetrize' the preceding paragraph. Indeed, it provides us with a group homomorphism $\widetilde{\phi}: \operatorname{Out}^{\tilde{\mathbf{x}}}(B) \rightarrow \operatorname{Out}(A)$ which assigns to $\bar{\tau} \in$ $\operatorname{Out}^{\tilde{\mathbf{X}}}(B)$ the unique element $\bar{\sigma} \in \operatorname{Out}(A)$ with ${ }^{\tau}\left(\widetilde{\mathbf{X}} \stackrel{L}{\otimes}_{A}-\right) \cong \widetilde{\mathbf{X}} \stackrel{L}{\otimes}_{A}{ }^{\sigma}(-)$, and we obtain $\operatorname{Im}(\widetilde{\phi}) \subseteq \operatorname{Out}^{\mathbf{X}}(A)$ as above. From the fact that $\mathbf{X}$ and $\widetilde{\mathbf{X}}$ are inverse to each other, we moreover derive that $\phi$ and $\widetilde{\phi}$ are inverse group isomorphisms between $\operatorname{Out}^{\mathbf{x}}(A)$ and $\operatorname{Out}^{\tilde{\mathbf{x}}}(B)$. In particular, $\operatorname{Im}(\phi)=\operatorname{Out}^{\tilde{\mathbf{x}}}(B)$. In view of part (2) of Proposition 15, finally, injectivity of $\phi$ guarantees that the canonical map $\pi: \mathcal{S}(\mathrm{X} e) / \mathcal{U} \rightarrow \mathcal{S}(\mathbf{X} e) / \mathcal{V}$ is the identity; in other words, we also have $\mathcal{U}=\mathcal{V}$. This completes the argument.

\section{ACKNOWLEDGEMENT}

The paper was prepared while the second-named author was visiting the University of California at Santa Barbara. He would like to thank the UCSB Mathematics Department for its hospitality. Moreover, he is grateful to the D.G.E.S. of Spain and the Fundación 'Séneca' of Murcia for their financial support.

\section{REFERENCES}

1. F.K. Anderson and K.R. Fuller, Rings and Categories of Modules, 2nd edition, SpringerVerlag, Berlin, 1992. MR 94i:16001

2. M. Auslander and S.O. Smalø, Preprojective modules over Artin algebras, J. Algebra 66 (1980), 61-122. MR 83a:16039

3. A. Borel, Linear Algebraic Groups, 2nd edition, Springer-Verlag, Berlin, 1991. MR 92d:20001

4. E. Cline, B. Parshall and L. Scott, Derived categories and Morita theory, J. Algebra 104 (1986), 397-409. MR 88a:16075

5. J.A. de la Peña, Tame algebras. Some fundamental notions, Universität Bielefeld. Ergänzungsreihe 95-010, 1995.

6. A. Fröhlich, The Picard group of noncommutative rings, in particular of orders, Trans. Amer. Math. Soc. 180 (1973), 1-45. MR 47:6751

7. F. Guil Asensio and M. Saorín, On automorphism groups induced by bimodules, Arch. Math. (Basel) 76 (2001), 12-19. CMP 2001:07

8. D. Happel, Triangulated Categories in the Representation Theory of Finite Dimensional Algebras, London Math. Soc. Lecture Note Series 119, Cambridge Univ. Press, Cambridge, 1988. MR 89e: 16035

9. B. Keller, Invariance and localization for cyclic homology of DG algebras, J. Pure Appl. Algebra 123 (1998), 223-273. MR 99c:16099

10. H. Kraft, Geometrische Methoden in der Invariantentheorie, Second ed., Vieweg, Braunschweig, 1985. MR 86j:14006

11. H. Lenzing and H. Meltzer, The automorphism group of the derived category for a weighted projective line, Comm. Algebra 28 (2000), 1685-1700. MR 2001a:16021

12. M. Linckelmann, Stable equivalences of Morita type for self-injective algebras and p-groups, Math. Zeitschr. 223 (1996), 87-100. MR 97j:20011

13. R. D. Pollack, Algebras and their automorphism groups, Comm. Algebra 17 (1989), 18431866. MR 90k:16037 
14. J. Rickard, Morita theory for derived categories, J. London Math. Soc. (2) 39 (1989), 436-456. MR 91b:18012

15. _ Derived equivalences as derived functors, J. London Math. Soc. (2) 43 (1991), 37-48. MR 92b:16043

16. R. Rouquier, Groupes d'automorphismes et équivalences stables ou dérivées, Preprint.

17. R. Rouquier and A. Zimmermann, Picard groups for derived module categories, Preprint.

18. J.L. Verdier, Catégories dérivées, état 0, in SGA $4 \frac{1}{2}$, Lecture Notes in Math. 569, SpringerVerlag, Berlin, 1977, pp. 262-311. MR 57:3132

19. D. Voigt, Induzierte Darstellungen in der Theorie der endlichen, algebraischen Gruppen, Lecture Notes in Math. 592, Springer-Verlag, Berlin, 1977. MR 58:5949

20. A. Yekutieli, Dualizing complexes, Morita equivalence and the derived Picard group of a ring, J. London Math. Soc. 60 (1999), 723-746. CMP 2000:11

Department of Mathematics, University of California, Santa Barbara, California 93106

E-mail address: birge@math.ucsb.edu

Departamento de Mátematicas, Universidad de Murcia, 30100 Espinardo-MU, Spain

E-mail address: msaorinc@fcu.um.es 